\title{
Data-Driven Ship Digital Twin for Estimating the Speed Loss caused by the Marine Fouling
}

\author{
Andrea Coraddu ${ }^{\mathrm{a}, *}$, Luca Oneto ${ }^{\mathrm{b}}$, Francesco Baldic ${ }^{\mathrm{c}}$, Francesca Cipollini ${ }^{\mathrm{b}}$, \\ Mehmet Atlar ${ }^{\mathrm{a}}$, Stefano Savio ${ }^{\mathrm{d}}$ \\ ${ }^{a}$ Naval Architecture, Ocean 85 Marine Engineering \\ Strathclyde University, Glasgow G1 1XW, UK \\ ${ }^{b}$ DIBRIS - University of Genova, Via Opera Pia 13, I-16145 Genova, Italy \\ ${ }^{c}$ École Polytechnique Fédérale de Lausanne, EnergyPolis, 1950 Sione \\ ${ }^{d}$ DITEN - University of Genova, Via Opera Pia 11a, I-16145 Genova, Italy
}

\begin{abstract}
Shipping is responsible for approximately the $90 \%$ of world trade leading to significant impacts on the environment. As a consequence, a crucial issue for the maritime industry is to develop technologies able to increase the ship efficiency, by reducing fuel consumption and unnecessary maintenance operations. For example, the marine fouling phenomenon has a deep impact, since to prevent or reduce its growth which affects the ship consumption, costly drydockings for cleaning the hull and the propeller are needed and must be scheduled based on a speed loss estimation. In this work a data driven Digital Twin of the ship is built, leveraging on the large amount of information collected from the on-board sensors, and is used for estimating the speed loss due to marine fouling. A thorough comparison between the proposed method and ISO 19030, which is the de-facto standard for dealing with this task, is carried out on real-world data coming from two Handymax chemical/product tankers. Results clearly show the effectiveness of the proposal and its better speedloss prediction accuracy with respect to the ISO 19030, thus allowing reducing the fuel consumption due to fouling.
\end{abstract}

\footnotetext{
*Corresponding Author

Email addresses: andrea.coraddu@strath.ac.uk (Andrea Coraddu), luca.oneto@unige.it (Luca Oneto), francesco.baldi@epfl.ch (Francesco Baldi), francesca.cipollini@edu.unige.it (Francesca Cipollini), mehmet.atlar@strath.ac.uk (Mehmet Atlar), stefano.savio@unige.it (Stefano Savio)
} 
Keywords: Hull and propeller maintenance, Fouling, Condition Based Maintenance, ISO 19030, Digital Twin, Data-Driven Models, Deep Learning.

\section{Introduction}

As awareness of climate change increases, new research results keep confirming that there is a need of fast, and strong action (IPCC, 2018). International maritime transport, while representing approximately $90 \%$ of global trade and the backbone of global economy, contributes to approximately $2.7 \%$ of the global anthropogenic carbon dioxide $\left(\mathrm{CO}_{2}\right)$ emissions (Smith et al. 2014). While this might appear a limited contribution, if current trends are not changed shipping will become one of the largest shares of global emissions (Anderson and Bows, 2012), since as of today, ships are still almost entirely powered by fossil fuels. While alternative fuels have shown to be promising (Gilbert et al., 2018), there is a need for ship energy systems to become more energy efficient (Lützen et al., 2017). As a result, sustainable shipping is recognised as one of the biggest challenges of the 21st century both for its contribution to $\mathrm{CO}_{2}$ emissions and to other pollutants (Schim van der Loeff et al., 2018).

In recent years, however, the International Maritime Organization (IMO) has officially adopted an initial strategy aiming at reducing Greenhouse Gases (GHG) emissions from shipping by 50\%, compared to 2008 levels, by 2050 , and to work towards phasing out them entirely by the end of the century (MEPC, 2018). As a consequence, developing new technologies able to both improve the design of the ships and to maintain their efficiency becomes a crucial issue (Deshpande et al. 2013). In this work, much attention was focused on the problem of keeping the ship as much efficient as possible by estimating the degradation state of its components, with the consequent performance loss and fuel consumption increase (Krozer et al., 2003). Broadly speaking, as far as propulsion systems are concerned, there are mainly three macro-components in a ship that can degrade: the main engine, the hull, and the propeller (Adland et al., 2018). Apart from the ordinary regular maintenance, the main engine degrades very slowly in time and related effects are only noticeable after years of operations (Calder, 1992). The hull and the

\footnotetext{
${ }^{1}$ http://www.ssi2040.org
} 
propeller, instead, are subject to marine fouling, that increases the frictional resistance of the parts moving through the water and, hence, decreases their efficiency (Adland et al., 2018). The effects of marine fouling can be clearly observed after just a few months of operations (Psaraftis and Kontovas, 2014; Demirel et al., 2017a). Marine fouling, or simply biofouling, is defined as the undesirable accumulation of microorganisms, algae, and animals on artificial surfaces immersed in seawater (Yebra et al., 2004; Lindgren et al., 2016). On the hull, the presence of fouling increases the roughness of the surface, hence increasing frictional resistance (Schultz, 2004; Kempf, 1937). On the propeller, the presence of fouling increases the roughness of the blade surface, thus requiring more power to maintain the same speed Atlar et al. 2002; Seo et al., 2016; Owen et al., 2018). Fouling represents the primary cause of hull (Candries et al., 2003) and propeller (Khor and Xiao, 2011) performance degradation.

Currently, shipping companies try to mitigate the problem of hull and propeller fouling by applying anti-fouling paints on the submerged surfaces and by regularly cleaning the hull (Lam and Lai, 2015). Despite their effectiveness, such methods have some drawbacks. In spite of their prime role and effectiveness in preventing fouling growth, depending on their types, antifouling paints can be expensive (e.g. non-biocidal Fouling Release type) and can be harmful to the marine environment (e.g. biocidal Self-Polishing types) (Carić et al., 2016). Moreover, the hull and the propeller are cleaned on the occasion of other dry-docking maintenance events, but this practice does not ensure an optimal scheduling of the cleaning procedures (Kjaer et al., 2018). Over a typical $4-5$ years sailing interval, inadequate hull and propeller performance is estimated to reduce the efficiency of the entire world fleet by $9-12 \%$ (CSC, 2011). This comes also as a consequence of the difficulty of identifying the actual contribution of fouling to the decrease in ship performance, and shipping companies have called for the establishment of a transparent and reliable standard for measuring hull and propeller performance (CSC, 2011). A reliable and effective planning of these activities should take into account the speed loss caused by the fouling, to find the optimal balance between efficiency and costs. For this reason an accurate estimation of the speed loss caused by fouling is needed (Schultz, 2007; Atlar et al., 2018).

However, providing a quantitative estimation of the speed loss associated to the fouling phenomenon is a challenging task (Demirel et al., 2017a b; Carchen et al., 2017). The latter depends on many factors, such as the speed 
and the draft of the ship, the sea state, the wind speed and direction, etc. Furthermore the accumulation of marine organisms on the hull is faster when a vessel is frequently in harbour, stationary, or in high-temperature tropical waters (Stevens, 1937).

The state-of-the-art approach for estimating the speed loss can be carried out by applying the standard ISO 19030 (ISO 19030-2, 2016) proposed by the International Organization for Standardization (ISO). The ISO 19030 prescribes methods for measuring changes in hull and propeller performance and it defines a set of relevant performance indicators for their maintenance, repair, and retrofit activities. Specifically, the ISO 19030 suggests comparing the measured performances with the ones obtained during sea trials in particular operating points. This comparison provides an indicator of the hull and propeller efficiency. A continuous monitoring of the efficiency provides a reliable estimation of the changes in the performances. Despite its simplicity and effectiveness, the ISO 19030 presents some limitations. The procedure requires filtering out operating points that are outside the prescribed boundaries, thus limiting the ability of the method to monitor the ship over a wide set of operating conditions (Koboević et al., 2018). Moreover, some corrections are needed to cope with the environmental disturbances (i.e. winds, waves, and currents). Unfortunately, these corrections require the use of complex fluid dynamics models or additional sea trials (ISO 19030-1, 2016; ISO 19030-2, 2016; ISO 19030-3, 2016).

Some attempts have been made to address the ISO 19030 limitations. Logan (2012) uses measurements of the propeller performance as efficiency indicators; however, this procedure requires the exclusion of many operating points to eliminate the effects of current, ship motions, rudder, and transients, with techniques similar to the ones reported in the ISO 19030 and with all their inconveniences. Bialystocki and Konovessis (2016) propose an operational approach for obtaining an accurate fuel consumption and speed curve, parametrised for the major influence factors, such as ship's draft and displacement, waves forces and directions, hull and propeller roughness. The proposed approach, similarly to the ISO 19030 procedure, relies on simplified corrections for environmental disturbances, draught, and speed. This applies also for the work proposed by Foteinos et al. (2017), whose method is based on a correction of measured data based on a physical model of the influence of wind and waves on ship performance.

While models based on the physical knowledge of the problem are wellestablished (Logan, 2012; ISO 19030-1, 2016), they often fail in predicting 
the effect of ship-specific and environmental phenomena. On the other hand, Data-Driven Models (DDMs) can easily take into account many phenomena thanks to the use of large amount of data with little, if no physical knowledge about the problem. DDMs have shown to be an effective tool for the solution of many problems in the shipping industry: condition based maintenance of the propulsion system (Cipollini et al., 2018), crash stop maneuvering performance prediction (Oneto et al., 2017a), operational profile prediction and optimisation (Coraddu et al., 2016), fuel consumption prediction and optimisation (Parlak et al., 2006). In particular, Neural Networks and Gaussian Process were employed to estimate the ship's fuel consumption efficiency in Pedersen and Larsen (2009) and Petersen et al. (2012), while in Radonjic and Vukadinovic (2015) a Neural Network Ensemble is exploited for towboat shaft power prediction. In Jonge (2017) many different supervised and unsupervised data-analytic models were adopted in order to investigate the relation between several vessel performance and environmental variables. Finally, in Leifsson et al. (2008) the performances of white, grey, and black box models for predicting the fuel consumption are tested.

Deep Learning techniques represent the state-of-the-art for dealing with data driven problems, despite its limitations. In particular, all the hidden parameters in Deep Learning framework need to be fine-tuned multiple times and are affected by the problem of local minima and slow convergence rate (Kasun et al., 2013). Recently some attempts have been made to overcome these limitations.

In particular, ELM (Cambria and Huang, 2013; Huang et al., 2015, 2006a) were introduced to overcome problems posed by back-propagation training algorithm (Huang, 2014, 2015; Ridella et al., 1997; Rumelhart et al., 1988): potentially slow convergence rates, critical tuning of optimisation parameters, and presence of local minima that call for multi-start and re-training strategies. The original ELM are also called Shallow ELM (SELM) because they have been developed for the single-hidden-layer feedforward neural networks (Huang et al., 2008, 2006b, 2004), and they have been generalised in order to cope with cases where ELM are not neuron alike. SELM were later improved to cope with problems intractable by shallow architectures (Bengio et al. 2013; Vincent et al., 2008, Zhou et al., 2015), by proposing various Deep ELM (DELM) built upon a deep architecture (Tissera and McDonnell, 2016; Oneto et al., 2017b), in order to make possible to extract features by a multilayer feature representation framework. In this work, the use of DELM is proposed for estimating the speed loss caused by the marine fouling effects 
on the ship hull and propeller, leveraging on the large amount of information collected from the on-board monitoring system sensors. Inspired by the ISO 19030 and supported by the evidence that DDMs can be much more accurate and effective than the physical ones, a DDM is proposed for predicting the speed of the ship, able to act as a "Digital Twin" (Glaessgen and Stargel, 2012) of the ship itself. The Digital Twin can be used to compute the deviation between the predicted performance and the actual one, namely the speed loss (Boschert and Rosen, 2016). It will be shown that the average drift in time of the speed loss can be exploited to accurately and effectively estimate the effects of the marine fouling on the ship performance, and thus program a more efficient hull and propeller cleaning scheduling. To this aim, they propose a two-phase approach:

(I) firstly, a DDM based Digital Twin is built, leveraging on the large amount of information collected from the on-board monitoring system sensors;

(II) secondly, the same model is applied in order to estimate the speed-loss of the ship and its drift.

Obviously the Digital Twin needs to be tuned on data collected during a period of time where the marine fouling is not present and for a time period wide enough to observe the ship in many operational and environmental conditions: data collection can start just after the launch of the ship (or after hull and propeller cleaning) and stop after some months of operations. Deep Learning techniques represent the state-of-the-art for dealing with Phase (I), For what concerns Phase (II), instead, it will be shown that the average behaviour of the speed loss between two maintenance events (where also hull and propeller cleaning is performed) is characterised by a clear drift, easily detectable with a robust regression (Zhao and Sun, 2010) in time of the predicted speed losses. Moreover, it will be shown, by means of the nonparametric statistical test of Kolmogorov-Smirnov (Smirnov, 1944), that the distribution of the speed loss before and after two maintenance events changes in a statistical significant way while, during the operations, such a distribution changes smoothly.

A comparison between the proposed method and the ISO 19030 on realworld data coming from two Handymax chemical/product tankers has been carried out and is presented in this work to show the effectiveness of the proposal.

The paper is organised as follows. Section 2 reports a general description 
of the two Handymax chemical/product tankers, and the monitoring system data considered. Section 3 presents a description of the standard application of ISO 19030. The proposed approach is described in Section 4. A comparison between the proposed method and the ISO 19030 on real-world data coming from the two vessels described in Section 2 is reported in Section 5. Finally, in Section 6, the conclusions of the paper are drawn.

For the benefit of the reader Table 1 containing all the adopted notation was added.

\section{Available Vessels and Data}

This section presents the two Handymax chemical/product tankers exploited, their data logging systems, and the available data adopted in the paper for comparing the proposed method and the ISO 19030, as far as the estimate of the speed loss caused by the marine fouling is concerned.

In this paper operational data available from two Handymax chemical/product tankers are used. The first vessel (V1) was designed and built for transporting chemicals and petroleum products up to $46764 D W T$ with a design speed of $15 \mathrm{knots}$. The vessel is $176.75 \mathrm{~m}$ long (between perpendicular) and $32.18 \mathrm{~m}$ wide, run by two four-stroke engines providing a total propulsive power of $7680 \mathrm{~kW}$. The second vessel (V2) is a tanker for chemicals and oil products up to $46067 D W T$ with a design speed of 15.5 knots. The vessel is $176.83 \mathrm{~m}$ long (between perpendicular) and $32.20 \mathrm{~m}$ wide, run by a two-stroke engine power of $8200 \mathrm{~kW}$. A conceptual representation of the propulsion system of the two vessels is shown in Figure 1, while their main features are presented in Table 2 .

The first vessel (V1) is equipped with two main engines (MaK 8M32C four-stroke Diesel engines rated $3840 \mathrm{~kW}$ ) and designed for operation at $600 \mathrm{rpm}$. The engines are connected to a gearbox that distributes the power between the controllable pitch propeller for propulsion and a shaft generator (rated $3200 \mathrm{~kW}$ ). Auxiliary power can also be generated by two auxiliary engines rated $682 \mathrm{~kW}$ each. Each main engine is equipped with exhaust gas boiler, that can be integrated with two auxiliary oil-fired boilers.

The second vessel (V2) is equipped with one main engine (MAN B\&W $6 \mathrm{~S} 50 \mathrm{MC}$ slow speed, two-stroke engine rated $8200 \mathrm{~kW}$ ) and designed for operation at $120 \mathrm{rpm}$. In this case, the auxiliary power is generated by three Diesel-generators rated $1176 k W$ each. As for V1, the main engine is equipped 
Table 1: Acronyms and Symbols

\begin{tabular}{|c|c|}
\hline Name & Meaning \\
\hline GHG & Greenhouse Gas \\
\hline DDM & Data-Driven Model \\
\hline ELM & Extreme Learning Machine \\
\hline SELM & Shallow Extreme Learning Machine \\
\hline DELM & Deep Extreme Learning Machine \\
\hline V1 & Vessel 1 \\
\hline $\mathrm{V} 2$ & Vessel 2 \\
\hline$\Delta_{i}$ & Difference between the i-th datum and the mean value \\
\hline$\sigma$ & Standard Deviation \\
\hline$N$ & Size of the dataset \\
\hline$v_{v}$ & Vessel speed \\
\hline$v_{w}$ & Wind speed \\
\hline$v_{\text {exp }}$ & Expected speed \\
\hline$v_{m}$ & Measured speed \\
\hline$\Delta_{P}$ & Power correction \\
\hline$R_{w}$ & Actual ship wind resistance \\
\hline$R_{0 w}$ & Ship wind resistance in calm water conditions \\
\hline$\eta_{p}$ & Actual propulsive efficiency in calm water conditions \\
\hline$\eta_{0 p}$ & Propulsive efficiency \\
\hline$P_{p}$ & Propulsive power \\
\hline$P_{p}^{\prime}$ & Corrected propulsive power \\
\hline$\rho_{a}$ & Air density \\
\hline$A_{t p}$ & Transverse projected area \\
\hline$C_{w}$ & Wind resistance coefficient \\
\hline$\psi_{w}$ & Wind relative direction \\
\hline$T$ & Draft \\
\hline$\delta$ & Trim \\
\hline $\mathrm{SL}_{\%}$ & Percentage speed loss \\
\hline $\boldsymbol{x}_{i}$ & Input vector belonging to $\mathcal{X} \subseteq \mathbb{R}^{d}$ \\
\hline$y_{i}$ & Output point belonging to $\mathcal{Y} \subseteq \mathbb{R}$ \\
\hline $\mathcal{D}_{n}$ & Dataset composed by $\left\{\left(\boldsymbol{x}_{i}, y_{i}\right)\right\}$ elements \\
\hline $\mathcal{L}_{n_{l}}^{r}$ & Training set composed by $n_{l}$ samples of $\mathcal{D}_{n}$ \\
\hline $\mathcal{V}_{n_{v}}^{r}$ & Validation Set composed by $n_{v}$ samples of $\mathcal{D}_{n}$ \\
\hline $\mathfrak{S}$ & Model mapping $\mathcal{X}$ to $\mathcal{Y}$ \\
\hline $\mathcal{H}$ & Set of hyperparameters \\
\hline $\mathcal{S}_{\mathcal{H}}$ & All sets of possible hyperparameters \\
\hline $\mathscr{A}_{\mathcal{H}}$ & Algorithm adopting the set of hyperparameters $\mathcal{H}$ \\
\hline $\mathcal{F}_{\mathcal{H}}$ & Set of models proposed by $\mathscr{A}_{\mathcal{H}}$ \\
\hline$f$ & Model in $\mathcal{F}_{\mathcal{H}}$ \\
\hline$\ell(f(\boldsymbol{x}), y)$ & Loss function \\
\hline$L(f)$ & Generalization Error \\
\hline$\widehat{L}(f)$ & Empirical Error \\
\hline$A$ & Activation Matrix \\
\hline$I_{[-\infty, x]}$ & Indicator Function \\
\hline$F(x)$ & Empirical cumulative probability distribution \\
\hline$D$ & Distance between two $F(x)$ \\
\hline
\end{tabular}




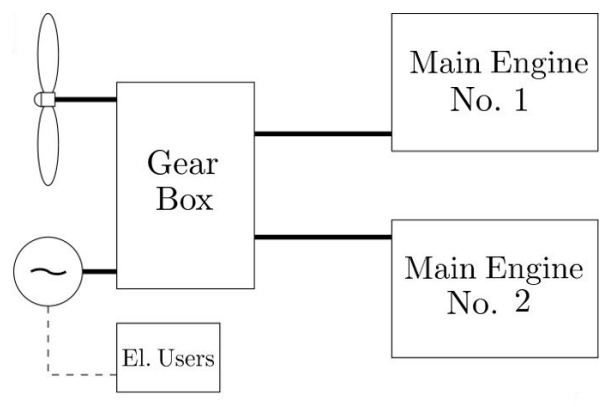

(a) V1

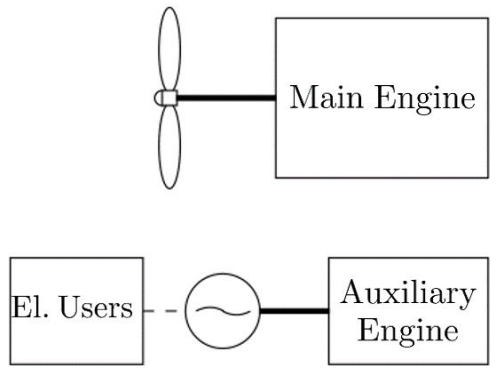

(b) V2

Figure 1: Conceptual representation of the propulsion systems of V1 and V2.

Table 2: Main features of V1 and V2 case studies.

\begin{tabular}{|l||r|r||r|r|}
\hline \hline \multicolumn{1}{|l||}{ Ship Feature } & \multicolumn{2}{c||}{ V1 } & \multicolumn{2}{c|}{ V2 } \\
\cline { 2 - 5 } & Value & Unit & Value & Unit \\
\hline \hline Deadweight & 46764 & {$[\mathrm{t}]$} & 46067 & {$[\mathrm{t}]$} \\
Design speed & 15 & {$[\mathrm{knots}]$} & 15.5 & {$[\mathrm{knots}]$} \\
Draft (summer SW) & 12.18 & {$[\mathrm{~m}]$} & 12.2 & {$[\mathrm{~m}]$} \\
Length between perpendicular & 176.75 & {$[\mathrm{~m}]$} & 176.83 & {$[\mathrm{~m}]$} \\
Breadth moulded & 32.18 & {$[\mathrm{~m}]$} & 32.20 & {$[\mathrm{~m}]$} \\
Main engines installed power & $3840 \times 2$ & {$[\mathrm{~kW}]$} & 8200 & {$[\mathrm{~kW}]$} \\
Auxiliary engines installed power & $682 \times 2$ & {$[\mathrm{~kW}]$} & $1176 \times 3$ & {$[\mathrm{~kW}]$} \\
Shaft generator power & 3200 & {$[\mathrm{~kW}]$} & & \\
Exhaust boilers steam generator & $750 \times 2$ & {$[\mathrm{~kg} / \mathrm{h}]$} & 1130 & {$[\mathrm{~kg} / \mathrm{h}]$} \\
Auxiliary boilers steam generator & $14000 \times 2$ & {$[\mathrm{~kg} / \mathrm{h}]$} & $14000 \times 2$ & {$[\mathrm{~kg} / \mathrm{h}]$} \\
Fuel consumption & 34.7 & {$[\mathrm{mt} / \mathrm{day}]$} & 31.8 & {$[\mathrm{mt} / \mathrm{day}]$} \\
\hline \hline
\end{tabular}


Table 3: Data collected from logging system of the two vessels.

\begin{tabular}{|l|r||l|r|}
\hline \hline Variable name & Unit & Variable name & Unit \\
\hline \hline Timestamp & {$[t]$} & Sea depth & {$[\mathrm{m}]$} \\
Latitude & {$\left[{ }^{\circ}\right]$} & Seawater temperature & {$\left[{ }^{\circ} \mathrm{C}\right]$} \\
Longitude & {$\left[{ }^{\circ}\right]$} & CPP set point & {$\left[{ }^{\circ}\right]$} \\
Main engines fuel consumption & {$[\mathrm{kg} / \mathrm{h}]$} & CPP feedback & {$\left[{ }^{\circ}\right]$} \\
Auxiliary engines power output & {$[\mathrm{kg} / \mathrm{h}]$} & Fuel density & {$\left[\mathrm{kg} / \mathrm{m}^{3}\right]$} \\
Shaft generator power & {$[\mathrm{kg} / \mathrm{h}]$} & Fuel temperature & {$\left[{ }^{\circ} \mathrm{C}\right]$} \\
Propeller shaft power & {$[\mathrm{kW}]$} & Ambient pressure & {$[\mathrm{bar}]$} \\
Propeller speed & {$[\mathrm{rpm}]$} & Humidity & {$[\%]$} \\
Ship draft (fore) & {$[\mathrm{m}]$} & Dew point temperature & {$\left[{ }^{\circ} \mathrm{C}\right]$} \\
Ship draft (aft) & {$[\mathrm{m}]$} & Shaft torque & {$[\mathrm{kNm}]$} \\
Draft port & {$[\mathrm{m}]$} & Rudder angle & {$\left[{ }^{\circ}\right]$} \\
Draft starboard & {$[\mathrm{m}]$} & Acceleration x direction & {$\left[\mathrm{m} / \mathrm{s}^{2}\right]$} \\
Relative wind speed & {$\left[\mathrm{m} / \mathrm{s}^{2}\right]$} & Acceleration y direction & {$\left[\mathrm{m} / \mathrm{s}^{2}\right]$} \\
Relative wind direction & {$\left[{ }^{\circ}\right]$} & Acceleration z direction & {$\left[\mathrm{m} / \mathrm{s}^{2}\right]$} \\
GPS heading & {$\left[{ }^{\circ}\right]$} & Roll & {$\left[{ }^{\circ}\right]$} \\
Speed over ground & {$[\mathrm{knots}]$} & Pitch & {$\left[{ }^{\circ}\right]$} \\
Speed through water & {$[\mathrm{knots}]$} & Yaw & $\left.{ }^{\circ}\right]$ \\
\hline \hline
\end{tabular}

with an exhaust gas boiler, that can be integrated with two auxiliary oil-fired boilers.

The two vessels are equipped with the same data logging system which is used by the company for both on board monitoring and land-based performance control. Table 3 summarises the available measurements from the continuous monitoring system. The original frequency of data acquisition by the monitoring system is equal to 1 point every 15 seconds. In order to provide easier data handling, the raw data are sent to the provider server, where they are processed to collect a set of 15 minutes averages. In this paper, the latter dataset was used for the application of the proposed method.

The available data of the two vessels have been collected in the time slots for V1, between the 21/03/2012 17:45:00 and the 03/10/2014 14:15:00, and for V2 between 01/05/2014 00:15:00 and 26/08/2016 14:15:00. Note that the data are characterised by many missing points due to failure in the data logging system, or maintenance, or stops of the vessels.

The two ships mainly operate according to a variable schedule, as far as both time spent at sea and ports visited are concerned. According to the market requirements, they operate over a wide range of routes (different operational and environmental conditions), thus making it hard to detect 
Table 4: Maintenance events for V1 and V2.

\begin{tabular}{|l|l|}
\multicolumn{2}{c|}{ V1 } \\
\hline \hline Date & Event \\
\hline \hline $21 / 03 / 2012$ & Vessel delivery \\
$29 / 10 / 2012$ & Propeller cleaning \\
$30 / 03 / 2013$ & Hull cleaning \\
$01 / 08 / 2013$ & Loss of the LOG speed measurement \\
$17 / 07 / 2014$ & Change from fixed-speed to variable-speed operations \\
\hline \hline
\end{tabular}

\begin{tabular}{|l|l|}
\hline \multicolumn{2}{c|}{ V2 } \\
\hline \hline Date & Event \\
\hline \hline $19 / 04 / 2014$ & Propeller polishing \\
$20 / 12 / 2014$ & Hull cleaning \\
$28 / 08 / 2015$ & Hull cleaning and Propeller polishing \\
$28 / 11 / 2015$ & Dry-docking \\
\hline
\end{tabular}

a small variation in ship performances. Figure 2 reports the distributions of the vessel speed, wind speed, trim, shaft torque, and power consumption retrieved from the available data for the two case studies. Based on these graphs, it is possible to state that the two available datasets can be considered good candidates for the validation of the proposed approach, being the collected data strongly affected by changes in operational and weather conditions. In fact, from Figure 2 it is possible to underline that although the two vessels present a similar speed distribution (see Figure 2(a)), they show a significant statistical difference in the draft and torque distributions, thus leading to a different fuel consumption. As a result, the two vessels can be considered for the validation of the proposed DELM method, providing a more complex and comprehensive validation scenario.

At last, Table 4 reports the recorded relevant maintenance events of the two vessels.

\section{ISO 19030 Procedure}

In order to check the effectiveness of the proposed method against a stateof-the-art approach, the procedure suggested by the ISO 19030 was implemented for monitoring hull and propeller performance (ISO 19030-1, 2016, ISO 19030-2, 2016; ISO 19030-3, 2016). In this section, the application procedure proposed by the ISO 19030 is presented from an operating point of 


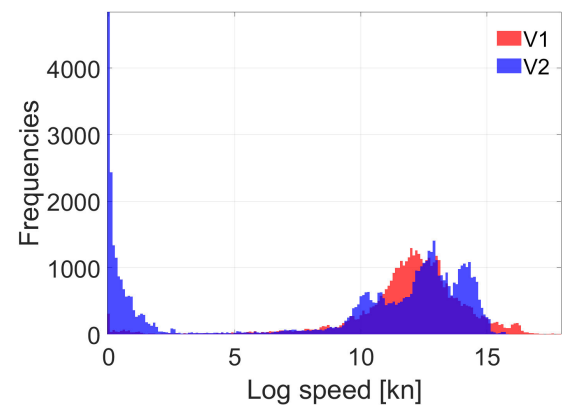

(a) Vessels Speed distribution

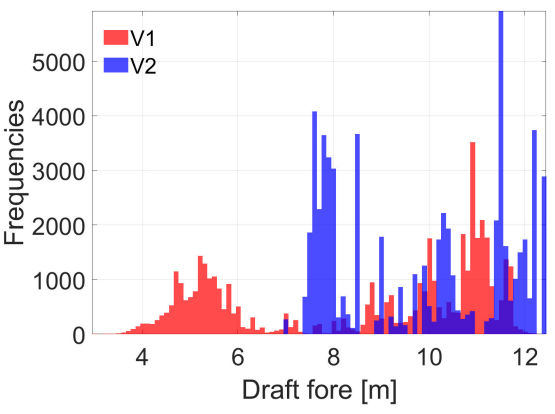

(c) Draft distribution (fore)

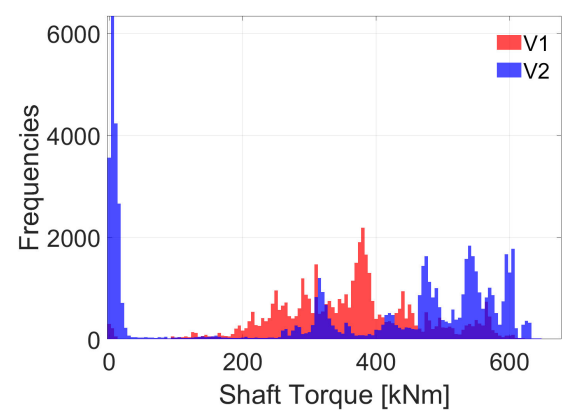

(e) Shaft Torque distribution

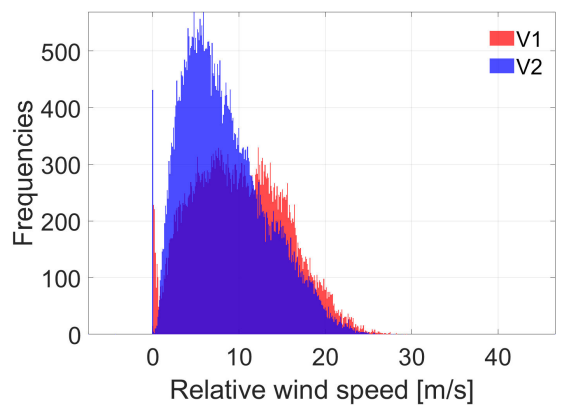

(b) Wind Speed distribution

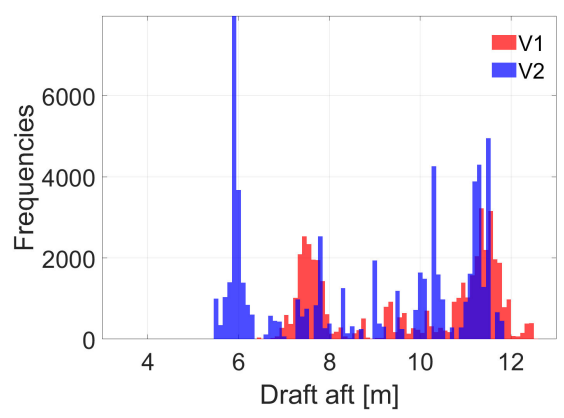

(d) Draft distribution (aft)

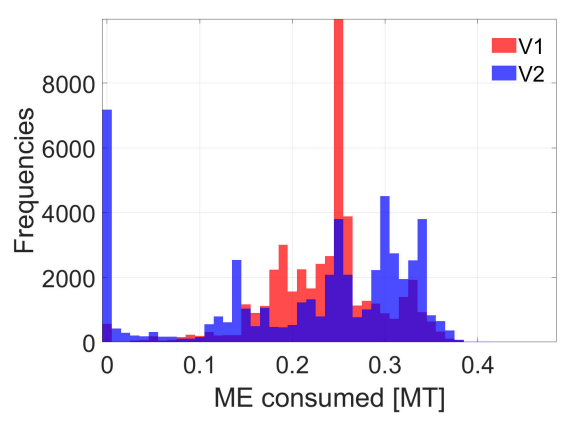

(f) Fuel Consumption distribution

Figure 2: Histograms containing the values distributions of V1 and V2 Vessels Speed, Wind Speed, Draft (fore and aft), Shaft Torque, and Fuel Consumption. 
view. More details are available in the reference documents ISO 15016:2015, 2015). The application of the ISO 19030 procedure, given the information collected from the data logging system as reported in Table 3, can be summarized in the following steps:

(I) Data filtering

(II) Correction for environmental factors

(III) Calculation of Performance Values (PVs)

(IV) Calculation of Performance Indicators (PIs)

Step (I) is performed by applying the Chauvenet's criterion (Chauvenet, 1863) to all measured variables, according to which a datum is to be considered an outlier if:

$$
\operatorname{erfc}\left(\frac{\Delta_{i}}{\sigma \sqrt{2}}\right) N<0.5
$$

where erfc is the complementary error function (Glaisher, 1871), $\Delta_{i}$ represents the difference between the i-th datum and the mean value over the dataset, $\sigma$ is the standard deviation of the variable of interest, and $N$ the size of the dataset. In addition, further filtering was applied considering outliers also points for which:

$$
v_{v}<8[\text { knots }], \quad\left|v_{w}\right|>8[\mathrm{~m} / \mathrm{s}]
$$

where $v_{v}$ and $v_{w}$ are the speed of vessel and wind respectively. The additional filtering on the ship speed was added in order to avoid numerical errors in the evaluation of the speed loss for low values in the denominator of Eq. (6), while the filter on the wind speed was added to filter out points with bad weather conditions, since the behaviour of the vessel in those conditions is strongly inconstant and unreliable. Step (II) included the power correction $\Delta_{P}$ based on measurements of wind speed and direction:

$$
\Delta_{P}=\left(R_{w}-R_{0 w}\right) v_{v}^{2} \frac{1}{\eta_{0 p}}+P_{p}\left(1-\frac{\eta_{p}}{\eta_{o p}}\right)
$$

where $R_{w}$ represents the ship's wind resistance due to relative wind, $R_{0 w}$ is the air resistance in no-wind conditions, $P_{p}$ is the propulsive power, $\eta_{p}$ its propulsive efficiency, and $\eta_{0 p}$ is the propulsive efficiency in calm condition. In absence of more accurate information, $\eta_{p}$ is set to 0.7 , as suggested by the ISO. The ship wind resistance $R_{r w}$ is computed as follows:

$$
R_{r w}=0.5 \rho_{a} v_{w}^{2} A_{t p} C_{w}\left(\psi_{w}\right)
$$


where $\rho_{a}$ is the air density, $A_{t p}$ is the transverse projected area, $C_{w}$ is the wind resistance coefficient, and $\psi_{w}$ is the wind relative direction. Eq. (4) is used for calculating both the actual and the reference wind resistance using the relative wind speed and the relative wind direction in the first case and the ship speed and head wind direction in the second case. The wind resistance coefficient is computed based on Fujiwara et al. (2006).

Step (III) involves the calculation of the percentage speed loss based on the corrected propulsion power. The expected speed $v_{\exp }$ is computed based on reference, clean-hull data interpolated starting from actual measurements of draft $(T)$ and trim $(\delta)$ :

$$
v_{\text {exp }}=f\left(P_{p}^{\prime}, T, \delta\right)
$$

where $P_{p}^{\prime}$ is the corrected power for accounting the effect of the draft and trim. This allows to compute the percentage speed loss SL\% as:

$$
\mathrm{SL}_{\%}=100 \frac{v_{m}-v_{e x p}}{v_{e x p}}
$$

where $v_{m}$ is the measured speed.

The speed loss is then used as performance value for the calculation of the different performance indicator in Step (IV). The ISO procedure suggests comparing the average value of the speed loss over a given period of time in order to average out uncertainties and statistically not-relevant fluctuations.

\section{Proposed Approach}

In this section the approach proposed in this paper is reported. As discussed in the introduction, the proposal is a two-phases approach:

(I) first a Digital Twin, based on a DDM, is built using a DELM and the data described in Section 2. The model exploits data collected during a suitable period of time when the marine fouling is not present and for a period long enough to observe the ship in different operational and environmental conditions (e.g. one can start the data collection just after the launch of the ship or its hull and propeller cleaning and stop after one or two months of operations);

(II) then the DDM is applied on a second set of data and the speed loss is computed. Subsequently, the drift in the average behaviour of the speed loss between two maintenance operations is studied, together 
with changes in its distribution using robust regression and statistical nonparametric test.

In the next section the two phases will be detailed.

\subsection{Phase (I)}

Phase (I) consists in mapping the task of predicting the speed of the vessel in a standard regression problem. In this framework (Vapnik, 1998), a set of data $\mathcal{D}_{n}=\left\{\left(\boldsymbol{x}_{1}, y_{1}\right), \cdots,\left(\boldsymbol{x}_{n}, y_{n}\right)\right\}$, where $\boldsymbol{x}_{i} \in \mathcal{X} \subseteq \mathbb{R}^{d}$ are the inputs and $y_{i} \in \mathcal{Y} \subseteq \mathbb{R}$ is an output, needs to be available. The goal is to identify the unknown model which maps inputs to outputs $\mathfrak{S}: \mathcal{X} \rightarrow \mathcal{Y}$ through an algorithm $\mathscr{A}_{\mathcal{H}}$ which chooses a model $f: \mathcal{X} \rightarrow \mathcal{Y}$ in a set of models $\mathcal{F}_{\mathcal{H}}$, defined by some hyperparameters $\mathcal{H}$.

In this specific case, the inputs and the outputs based on the available measurements reported in Table 3 are identified. In particular, Table 5 reports the chosen subset of features of the monitoring system adopted as input and output features for the proposed model. It is worth noting that the selected features provide the model with a good representation of the vessel's propulsion system, its motion and the weather conditions (Coraddu et al., 2015; Petersen et al., 2012). With respect to Table 3, Table 5 discards all those features which are duplicated, not necessary, or trivially related to the speed prediction.

The accuracy of $f$ in representing the unknown system $\mathfrak{S}$ is measured with a prescribed loss function $\ell: \mathcal{Y} \times \mathcal{Y} \rightarrow[0, \infty)$. Since the considered problem is a regression one, the most suited loss function is the squared one $\ell(f(\boldsymbol{x}), y)=[f(\boldsymbol{x})-y]^{2}$ (Rosasco et al., 2004). Then the generalization error of $f$, namely the true error of $f$, can be defined as

$$
L(f)=\mathbb{E}_{(\boldsymbol{x}, y)} \ell(f(\boldsymbol{x}, y)) .
$$

Obviously $L(f)$ cannot be computed but its empirical estimator, the empirical error, can be derived

$$
\widehat{L}(f)=\frac{1}{n} \sum_{i=1}^{n} \ell\left(f\left(\boldsymbol{x}_{i}, y_{i}\right)\right) .
$$

As far as the algorithm $\mathscr{A}_{\mathcal{H}}$ is concerned, in this paper the DELM is exploited, as described in Section 1. DELM are the evolution of the SELM for the purpose of creating an algorithm able to both learn new features from 
Table 5: Input and Output of the Digital Twin for Speed Prediction.

\begin{tabular}{|l|l|}
\hline \hline INPUT VARIABLES & OUTPUT VARIABLE \\
\hline \hline Latitude & \\
Longitude & \\
Main engines fuel consumption & \\
Auxiliary engines power output & \\
Shaft generator power & \\
Propeller shaft power & \\
Propeller speed & \\
Ship draft (fore) & \\
Ship draft (aft) & \\
Draft port & \\
Draft starboard & \\
Sea depth & \\
Relative wind speed & \\
Relative wind direction & \\
GPS heading & Speed through water \\
Sea water temperature & \\
CPP set point & \\
CPP feedback & \\
Fuel density & \\
Fuel temperature & \\
Ambient pressure & \\
Humidity & \\
Dew point temperature & \\
Shaft torque & \\
Rudder angle & \\
Acceleration x direction & \\
Acceleration y direction & \\
Acceleration z direction & \\
Roll & \\
Pitch & \\
Yaw & \\
\hline \hline
\end{tabular}


the available raw variables and create a regression model. For this reason, in order to understand the DELM, first the definition of SELM has to be recalled.

SELM were originally developed for the single-hidden-layer feedforward neural networks

$$
f(\boldsymbol{x})=\sum_{i=1}^{h} w_{i} g_{i}(\boldsymbol{x}) .
$$

where $g_{i}: \mathbb{R}^{d} \rightarrow \mathbb{R}, i \in\{1, \cdots, h\}$ is the hidden-layer output corresponding to the input sample $\boldsymbol{x} \in \mathbb{R}^{d}$, and $\boldsymbol{w} \in \mathbb{R}^{h}$ is the output weight vector between the hidden layer and the output layer. In this case, the input layer has $d$ neurons and connects to the hidden layer (having $h$ neurons) through a set of weights $W \in \mathbb{R}^{h \times(0, \cdots, d)}$ and a nonlinear activation function ${ }^{2}, \varphi: \mathbb{R} \rightarrow \mathbb{R}$. Thus, the $i$-th hidden neuron response to an input stimulus $\boldsymbol{x}$ is:

$$
g_{i}(\boldsymbol{x})=\varphi\left(W_{i, 0}+\sum_{j=1}^{d} W_{i, j} x_{j}\right) .
$$

In SELM, the parameters $W$ are set randomly. A vector of weighted links, $\boldsymbol{w} \in \mathbb{R}^{h}$, connects the hidden neurons to the output neuron without any bias. The overall output function of the network (see Figure 3) is:

$$
f(\boldsymbol{x})=\sum_{i=1}^{h} w_{i} \varphi\left(W_{i, 0}+\sum_{j=1}^{d} W_{i, j} x_{j}\right)=\sum_{i=1}^{h} w_{i} \varphi_{i}(\boldsymbol{x}) .
$$

It is convenient to define an activation matrix, $A \in \mathbb{R}^{n \times h}$, such that the entry $A_{i, j}$ is the activation value of the $j$-th hidden neuron for the $i$-th input pattern. The $A$ matrix is:

$$
A=\left[\begin{array}{ccc}
\varphi_{1}\left(\boldsymbol{x}_{1}\right) & \cdots & \varphi_{h}\left(\boldsymbol{x}_{1}\right) \\
\vdots & \ddots & \vdots \\
\varphi_{1}\left(\boldsymbol{x}_{n}\right) & \cdots & \varphi_{h}\left(\boldsymbol{x}_{n}\right)
\end{array}\right]
$$

In the SELM model the weights $W$ are set randomly and are not subject to any adjustment, and the quantity $\boldsymbol{w}$ in Eq. (11) is the only degree of freedom.

\footnotetext{
${ }^{2}$ In this work the tanh function was adopted as suggested in the original work of Huang et al. (2004), nevertheless using other activation functions such as the sigmoidal one does not really affect the final performance.
} 


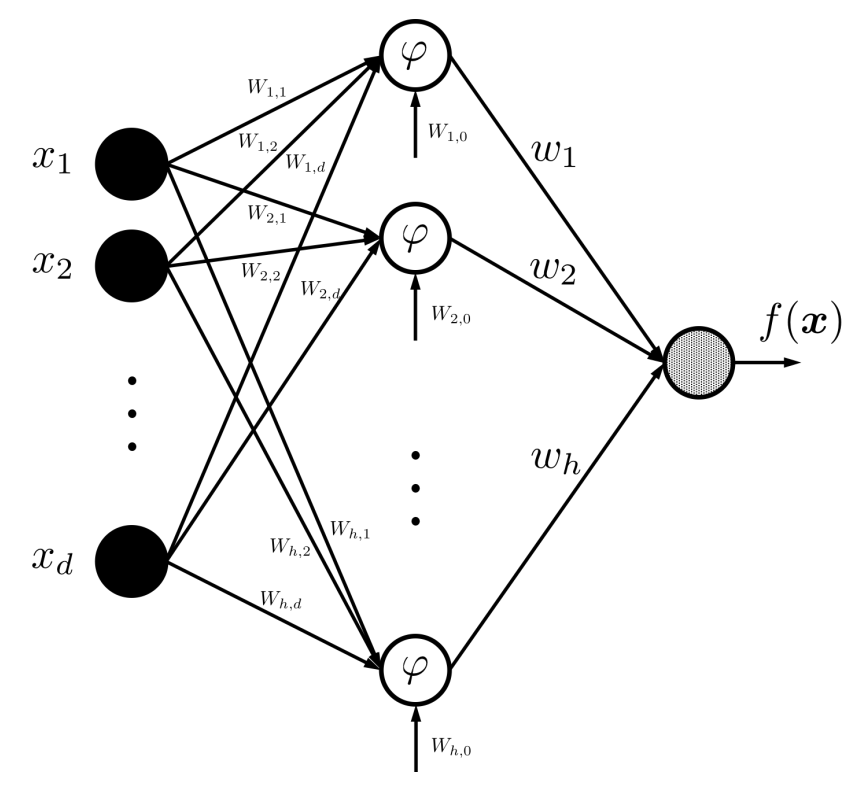

Figure 3: SELM structure.

Hence, the training problem reduces to a simple Regularized Least Squares (RLS) problem (Caponnetto and De Vito, 2007):

$$
\boldsymbol{w}^{*}=\arg \min _{\boldsymbol{w}}\|A \boldsymbol{w}-\boldsymbol{y}\|^{2}+\lambda\|\boldsymbol{w}\|^{2}
$$

where $\lambda \in[0, \infty)$ is a hyperparameter that must be tuned during the Model Selection (MS) phase Oneto (2018), since it balances the trade off between accuracy complexity of the model measures with the square loss and the $L 2$ regularizer respectively. Consequently, the vector of weights $\boldsymbol{w}^{*}$ is then obtained as follows:

$$
\boldsymbol{w}^{*}=\left(A^{T} A+\lambda I\right)^{+} A^{T} \boldsymbol{y}
$$

where $I \in \mathbb{R}^{h \times h}$ is an identity matrix and $(\cdot)^{+}$is the Moore-Penrose matrix pseudoinverse. Note that $h$, the number of hidden neurons, is another hyperparameter that needs to be tuned based on the problem under exam. Note also that other regularizers can be exploited (e.g. sparse regularizers (Tibshirani, 1996; Zou and Hastie, 2005)).

Due to its shallow architecture, feature learning using SELM may not be effective even when $h$ is large. Since feature learning is often useful to improve the accuracy of the final model, multilayer (deep) solutions are usually needed. In Kasun et al. (2013); Tang et al. (2016) multilayer learning 


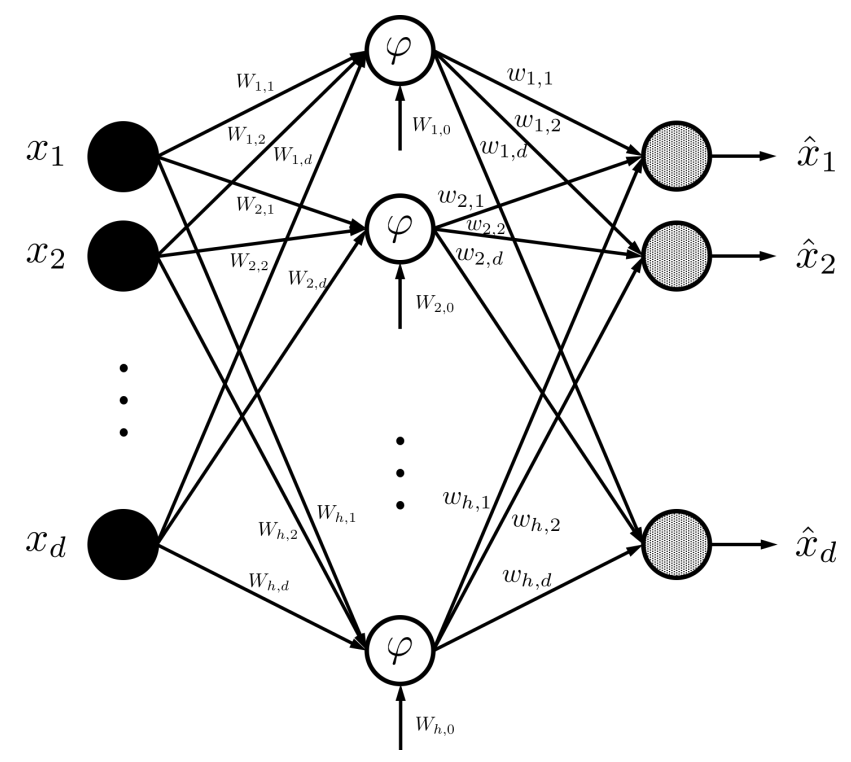

Figure 4: DELM AE block.

architectures are developed using ELM-based autoencoder (AE) as its building block (see Figure 4), which results in a sort of DELM. At each layer $i$ of the $l$ layers, each one composed of $h_{i \in\{1, \cdots, l\}}$ neurons, the DELM tries to reconstruct the input data and the outputs of the previous layer are used as the inputs of the next one. Basically, instead of having just one output, a series of outputs $\hat{x}_{j}$ with $j \in\{1, \cdots, d\}$ is obtained such that

$$
\hat{x}_{j}=f_{j}(\boldsymbol{x})=\sum_{i=1}^{h} w_{i, j} \varphi\left(W_{i, 0}+\sum_{j=1}^{d} W_{i, j} x_{j}\right)=\sum_{i=1}^{h} w_{i, j} \varphi_{i}(\boldsymbol{x}),
$$

where $w_{i, j}$ with $i \in\{1, \cdots, h\}$ are found with the same approach of SELM. Before the supervised regularized least mean square optimisation, the encoded outputs are directly fed into the last layer for decision-making, without random feature mapping. Differently from SELM, DELM do not require fine-tuning for the entire system and consequently the training speed can be much faster than the traditional back propagation based Deep Learning. Training the DELM is equivalent to training many SELM. Consequently, it is possible to take advantage of a deep architecture by exploiting only the optimisation tools presented for the SELM.

Note that the DELM is characterized by many hyperparameters: the number of layers, the number of nodes per layer, and the regularization 
coefficient: $\mathcal{H}=\left\{l, h_{1}, \cdots, h_{l}, \lambda\right\} . \mathcal{H}$ must be carefully tuned in order to obtain the smallest generalization error of the final model. For this purpose a MS phase needs to be performed (Oneto, 2018). In this work the nonparametric Bootstrap (BTS) approach is employed as it is a frequently adopted state-of-the-art MS method in the family of the resampling methods. Resampling methods derive their name from the procedure of resampling once or many $\left(n_{r}\right)$ times, with or without replacement, the original dataset $\mathcal{D}_{n}$, in order to build two independent datasets called training, and validation sets, respectively $\mathcal{L}_{n_{l}}^{r}$ and $\mathcal{V}_{n_{v}}^{r}$, with $r \in\left\{1, \cdots, n_{r}\right\}$. Note that $\mathcal{L}_{n_{l}}^{r} \cap \mathcal{V}_{n_{v}}^{r}=\oslash, \mathcal{L}_{n_{l}}^{r} \cup \mathcal{V}_{n_{v}}^{r}=\mathcal{D}_{n}$. Then, in order to perform the MS phase and select the best combination of the hyperparameters $\mathcal{H}$ in a set of possible ones $\mathcal{S}_{\mathcal{H}}=\left\{\mathcal{H}_{1}, \mathcal{H}_{2}, \cdots\right\}$ for the algorithm $\mathscr{A}_{\mathcal{H}}$, the following procedure has to be applied:

$$
\mathcal{H}^{*}: \min _{\mathcal{H} \in \mathcal{S}_{\mathcal{H}}} \frac{1}{n_{r}} \sum_{r=1}^{n_{r}} \frac{1}{n_{v}} \sum_{(\boldsymbol{x}, y) \in \mathcal{V}_{n_{v}}^{r}} \ell\left(\mathscr{A}_{\mathcal{H}, \mathcal{L}_{n_{l}}^{r}}(\boldsymbol{x}), y\right),
$$

where $\mathscr{A}_{\mathcal{H}, \mathcal{L}_{n_{l}}^{r}}$ is a model built with the algorithm $\mathscr{A}_{\mathcal{H}}$ trained with $\mathcal{L}_{n_{l}}^{r}$. Since the data in $\mathcal{L}_{n_{l}}^{r}$ are independent with respect to the ones in $\mathcal{V}_{n_{v}}^{r}, \mathcal{H}^{*}$ should achieve low error rates on a data set which is different from the one used for training purposes. BTS differentiates from the other resampling methods since $n_{l}=n$ and since $\mathcal{L}_{n_{l}}^{r}$ is sampled with replacement from $\mathcal{D}_{n}$. Note that $\mathcal{V}_{n_{v}}^{r}=\mathcal{D}_{n} \backslash \mathcal{L}_{n_{l}}^{r}$.

\subsection{Phase (II)}

Once the DELM based Digital Twin has been built, it is possible to apply it to the rest of the data in order to estimate the expected speed $v_{\text {expected }}$ and compare it with the measured one $v_{\text {measured }}$ for the purpose of computing the percentage speed loss $\mathrm{SL}_{\%}$. Note that this can be done both in a data driven way (see Section 4.1) and with the ISO 19030 (see Section 3).

The result of this process is a series of values in time representing the trend of the percentage speed loss

$$
\mathrm{SL}_{\%}(t), \quad t \in\left\{t_{1}, t_{2}, \cdots\right\}, \quad t_{1}<t_{2}<\cdots .
$$

The time series obtained with the DELM are referred as $\mathrm{SL}_{\%}^{\mathrm{DELM}}(t)$, while $\mathrm{SL}_{\%}^{\mathrm{ISO}}(t)$ are the one obtained with the ISO 19030.

The mentioned time series can be studied in two ways. 
In the first case the drift in the behaviour of the $\mathrm{SL}_{\%}(t)$ between two propeller and/or hull cleaning events was studied, by finding the best linear regressor for the speed loss percentage. Note that the time series computed from the data, as also observed by the ISO 19030, is characterized by uncertainties and irrelevant statistically fluctuations. For this reason, instead of applying a simple RLS, the robust regression developed in Zhao and Sun (2010) has been used. The idea of the robust regression applied to this case is quite simple. Firstly the regressor function had to be defined, in this case a linear regressor in time $g(t)=a t+b$ with $a, b \in \mathbb{R}$. Then instead of minimizing the mean square error, the following costs have been minimized

$$
a^{*}, b^{*}=\arg \min _{a, b \in \mathbb{R}} \sum_{t \in\left\{t_{1}, t_{2}, \cdots\right\}} \max \left[\min \left[a t+b-\mathrm{SL}_{\%}(t), \hat{\epsilon}\right], \check{\epsilon}\right]
$$

where $\hat{\epsilon}$ and $\check{\epsilon}$ are hyperparameters that needs to be tuned. Note that, basically, robust regression exploits a loss function which does not take into account too small or too large errors. Unfortunately, this loss is nonconvex and in Zhao and Sun (2010) a method for facing this issue is proposed. The robust regression allows obtaining results which are not affected by the high number of outliers observed in the time series (see Section 5).

In the second case, the automatic identification of changes in time of the distribution of the percentage speed loss was tried, in order to check if those changes were in correspondence to maintenance activities, and testify the quality of the estimated speed loss. For this purpose Kolmogorov-Smirnov test (Smirnov, 1944) has been adopted. This nonparametric statistical test can be exploited to check if two different data samples of data are derived from the same probability distribution. The Null Hypothesis is that the two samples belong to the same distribution. Then the test tries to quantify the distance between the distributions of the two samples and, if the distance is greater than a specific threshold, the hypothesis is rejected. The distance between the two samples $S^{A}=\left\{\mathrm{SL}_{\%}(t-\Delta), \cdots, \mathrm{SL}_{\%}(t)\right\}$ and $S^{B}=\left\{\mathrm{SL}_{\%}(t), \cdots, \mathrm{SL}_{\%}(t+\Delta)\right\}$, is computed exploiting the empirical cumulative probability distribution $F^{j}(x)$ of the two different samples, with $j \in A, B$ which is defined as:

$$
F_{j}(x)=\frac{1}{n} \sum_{i=1}^{n} I_{S_{i}^{j} \leq x}
$$

where $I_{[-\infty, x]}\left(S_{i}^{j}\right)$ is the indicator function, equal to 1 if $S_{i}^{j} \leq x$ and equal to 0 otherwise. The distance $D$ between $F^{A}(x)$ and $F^{B}(x)$ is computed adopting 
the following metric:

$$
D=\sup _{-\infty<x<+\infty}\left|F^{A}(x)-F^{B}(x)\right|
$$

For the analysis carried out in this paper, the maximum $D$ value tolerable to refuse the hypotheses was set at $95 \%$. The test was applied by comparing subsequent non-overlapping time windows of 30 days, in order to detect a broad variation in the series. It is worth noting that if the test is rejected, then a major change in the distribution of the error has occurred, thus indicating that the state of the vessel has abruptly changed.

\section{Results and discussion}

In this section, the available data described in Section 2 are exploited for the purpose of making a detailed comparison between the results obtained through the state-of-art ISO 19030 procedure, and the ones achieved via the DELM model. In particular, the properties of the speed losses estimated with the two models for both V1 and V2 are compared. The ISO 19030 model is built exploiting the procedure described in Section 3, while the DELM model is built adopting data for two months of historical data, in the proximity of a ship hull and propeller cleaning (for V1 the time slot between the $01 / 07 / 2012$ and the 15/09/2012, and for V2 the one between the 15/01/2016 and the 15/06/2016), using the procedure described in Section 4.1. Moreover, as far as the MS phase is concerned, the best set of hyperparameters was searched in

$$
\mathcal{S}_{\mathcal{H}}=\left\{\{1,2,4,8,16,32\} \times 10^{\{1,2,3,4\}} \times \cdots \times 10^{\{1,2,3,4\}} \times\left\{10^{-6}, 10^{-5.5}, \cdots, 10^{4}\right\}\right\} .
$$

As results will show, despite the accuracy and reliability of the sensors measurements collected on-board cannot be ensured (Coraddu et al., 2017), the proposed DELM allows the identification of clear drift in the performance of the vessel compared to the ISO 19030 procedure.

\subsection{Distributions of DELM and ISO 19030 Estimated Percentage Speed Loss}

In this section the histograms of the percentage speed losses computed with ISO 19030 and DELM approaches are proposed and discussed. In Figure 5 the histograms of the percentage speed losses for V1 and V2 are reported. From the histograms it is possible to observe that: 


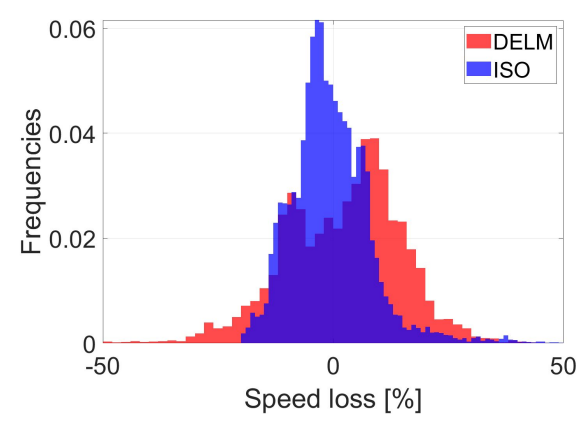

(a) V1

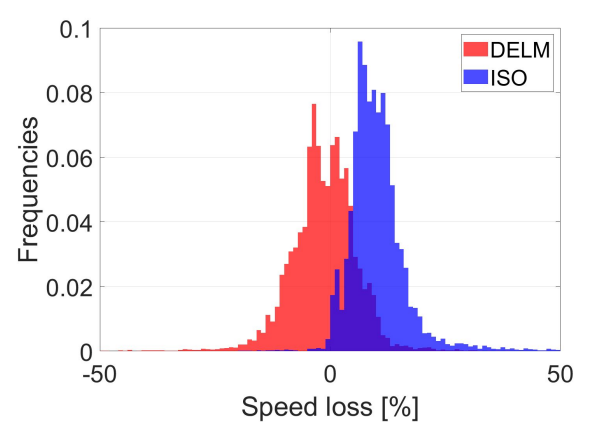

(b) V2

Figure 5: Histograms of DELM and ISO 19030 Estimated Percentage Speed Loss.

- as expected, the variance of the distribution of the percentage speed losses is lager for the DELM model with respect to the one of the ISO 19030. This is caused by the fact that the ISO 19030 filters out a large amount of data points, only keeping those for which the application of the method is more reliable (see Section 3). On the other hand, the DELM model exploits all the available data points corresponding to a larger variety of operational conditions;

- the average of the distribution of the speed loss is not always centered on a positive value, due to the fact that the data used for training the DELM and the parameters used for the ISO 19030 do not correspond to a perfect clean state, as it would be required for creating a perfect digital twin (as shown later, this problem does not affect the quality of the final results);

- the results obtained by the two models are, at least qualitatively, in an overall good agreement (quantitative assessment will be discussed in the next section).

\subsection{Scatterplot of DELM and ISO 19030 Estimated Percentage Speed Loss}

In order to better quantify the agreement between the DELM and the ISO 19030 models, Figure 6 reports the scatterplot of the DELM and the ISO 19030 estimated percentage speed loss for V1 and V2. From Figure 6 it is possible to observe that the speed loss predicted by the DELM and the ISO 19030 methods are positively correlated (particularly for V2), thus demonstrating that the prediction achievable by the proposed DELM approach is consistent with the state-of-the-art. 


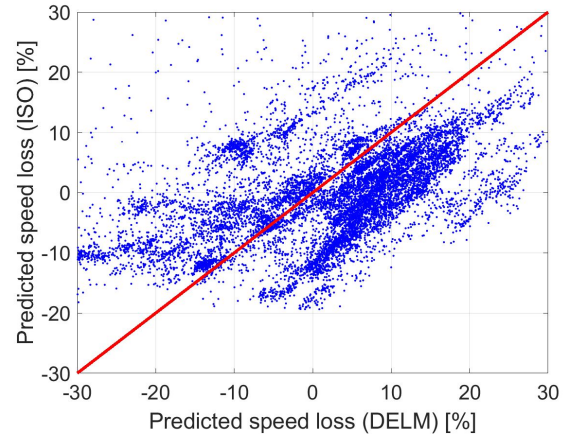

(a) V1

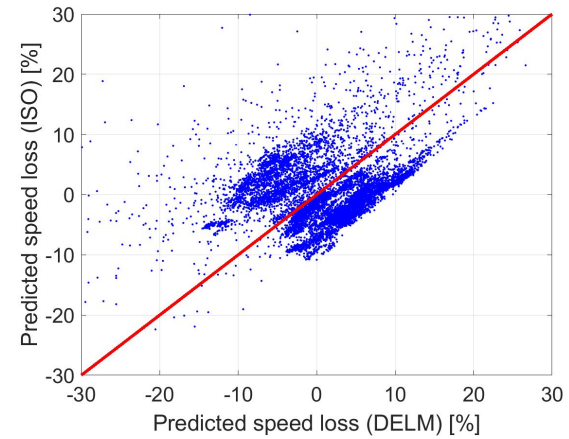

(b) V2

Figure 6: Scatterplot of the DELM and the ISO 19030 Estimated Speed Loss Percentages.

\subsection{Drift in DELM and ISO 19030 Estimated Percentage Speed Loss}

This section reports the analysis of the drift in DELM and ISO 19030 estimated percentage speed loss between two consecutive hull and propeller cleaning events, carried out with the linear robust regression described in Section 4.2 .

Figures 7 and 8 report the results for V1 and V2 respectively. Those results clearly show the higher level of reliability of the prediction achieved by the DELM method against the ISO 19030 one.

In both vessels, the linear trend for the speed loss calculated by the ISO 19030 method shows large variations between different maintenance intervals (particularly for V1, but also for V2). In addition, in some intervals between two consecutive hull and propeller cleaning operations, the trend in the estimated percentage speed loss using the ISO 19030 method is negative. These results do not agree with the physical basis of the fouling phenomenon, and suggest that, in the case presented in this paper, the application of the ISO can lead to inaccurate results.

On the contrary, as far as DELM is concerned, Figures 7 and 8 clearly show trends that are always physically plausible. Model drift behavior between different cleaning intervals is now consistent with the one characterizing a ship that operates in conditions, on average, similar over time.

\subsection{Changes in Time of DELM and ISO 19030 Estimated Percentage Speed Loss Distribution}

This section reports the analysis of the changes in time of DELM and 


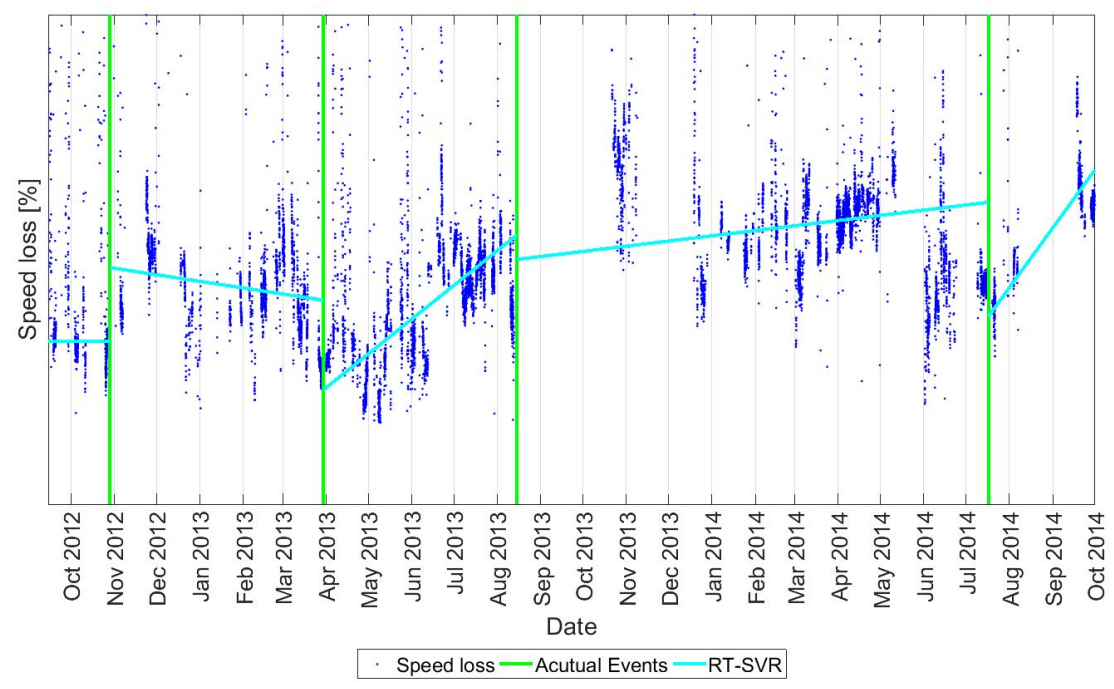

(a) ISO 19030

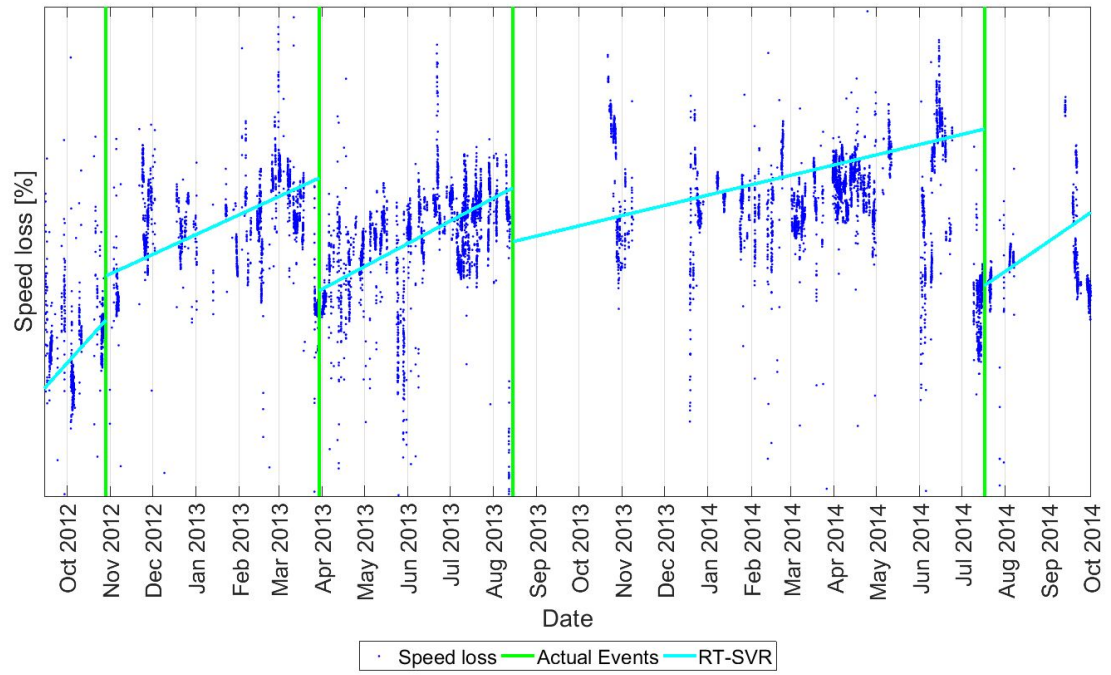

(b) DELM

Figure 7: Linear Robust Regression on the Speed Loss Percentages between two consecutive Hull and Propeller Cleaning Events for V1. 


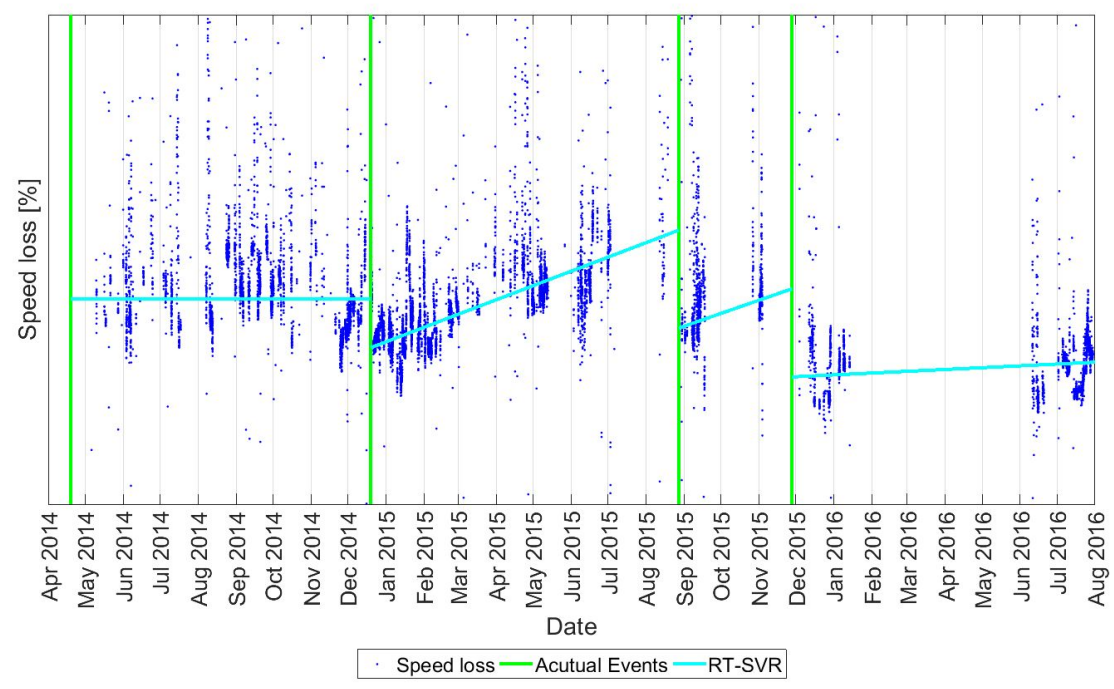

(a) ISO 19030

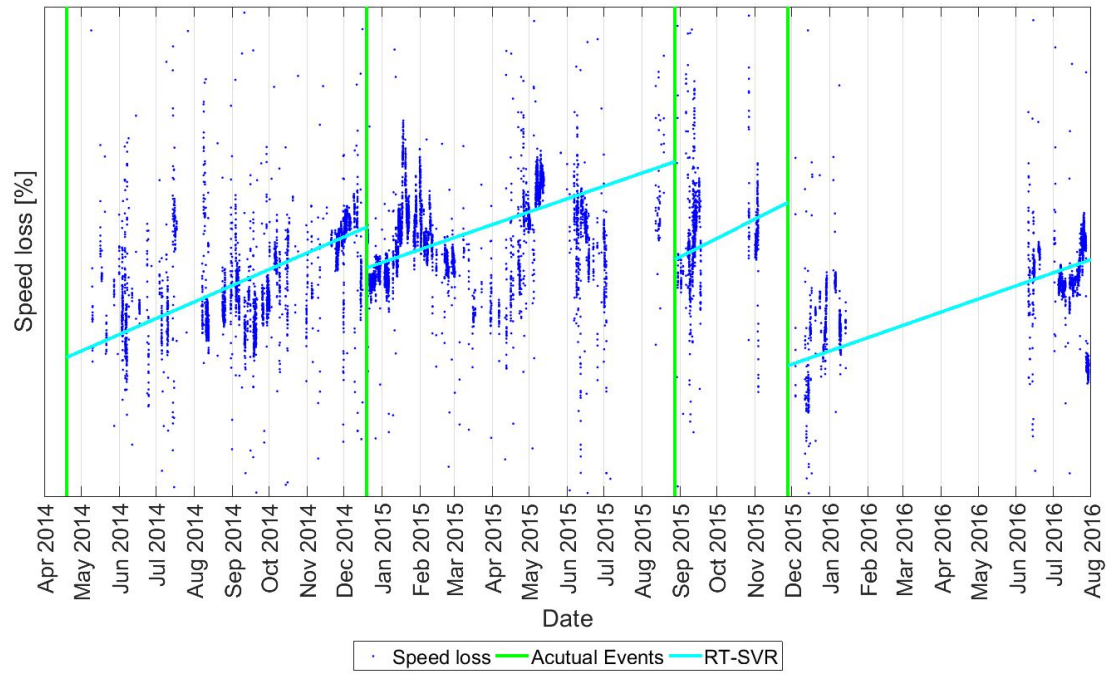

(b) DELM

Figure 8: Linear Robust Regression on the Speed Loss Percentages between two consecutive Hull and Propeller Cleaning Events for V2. 
ISO 19030 estimated percentage speed loss distributions, carried out with the nonparametric statistical test of Kolmogorov-Smirnov as described in Section 4.2.

Figures 9 and 10 report the results for V1 and V2 respectively. Those figures tesfify the higher level of reliability of the DELM method against the ISO 19030 one.

In both vessels there is no statistically meaningful changes in the distribution of the speed losses estimated with the ISO 19030, and just in a few cases the Kolmogorov-Smirnov test detects a change in correspondence to an actual hull and propeller cleaning event (see Table 4).

On the contrary, when the same method is applied to the speed losses estimated with the DELM approach, the Kolmogorov-Smirnov test detects all the changes in correspondence or close to an actual hull and propeller cleaning event.

From the obtained results, it is possible to firmly conclude that the proposed method based on DELM can provide a more accurate estimation of the changes in performance due to hull and propeller marine fouling with respect to the ISO 19030 based model.

\subsection{Significance of the results}

With this paper a new method for estimating the effect of fouling on ship performance is proposed. As showed in the previous sections, this method represents an improvement compared to ISO standards, while only requiring operational data as input (hence no model tests, ship design data, etc.). The need for an efficient calculation method for the effect of fouling on ship performance has been highlighted by many actors in shipping, both for economical and for environmental reasons (CSC, 2011).

The speed loss calculated using the linear robust regression as showed in Figures 7 and 8 provides an accurate picture of the status of the hull and propeller fouling at a specific point in time. This information could be used effectively to optimize the scheduling of maintenance events. Today, hull and propeller cleaning are performed at fixed intervals, or in correspondence of other maintenance events (e.g. dry-docking). In practice, they could be performed more or less often depending on the actual status of the hull and propeller, according to methods based on the minimization of costs, fuel consumption, and emissions.

Based on a similar principle, the method could be used as a mean for the critical evaluation of interventions directed towards the limitation of fouling 


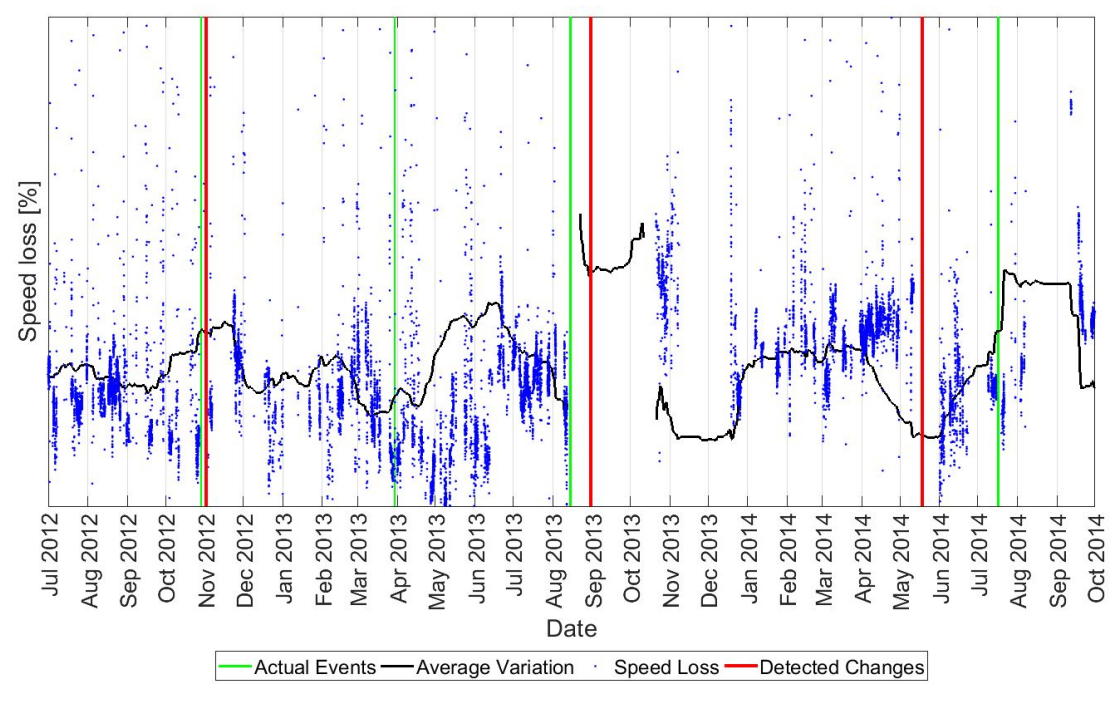

(a) ISO 19030

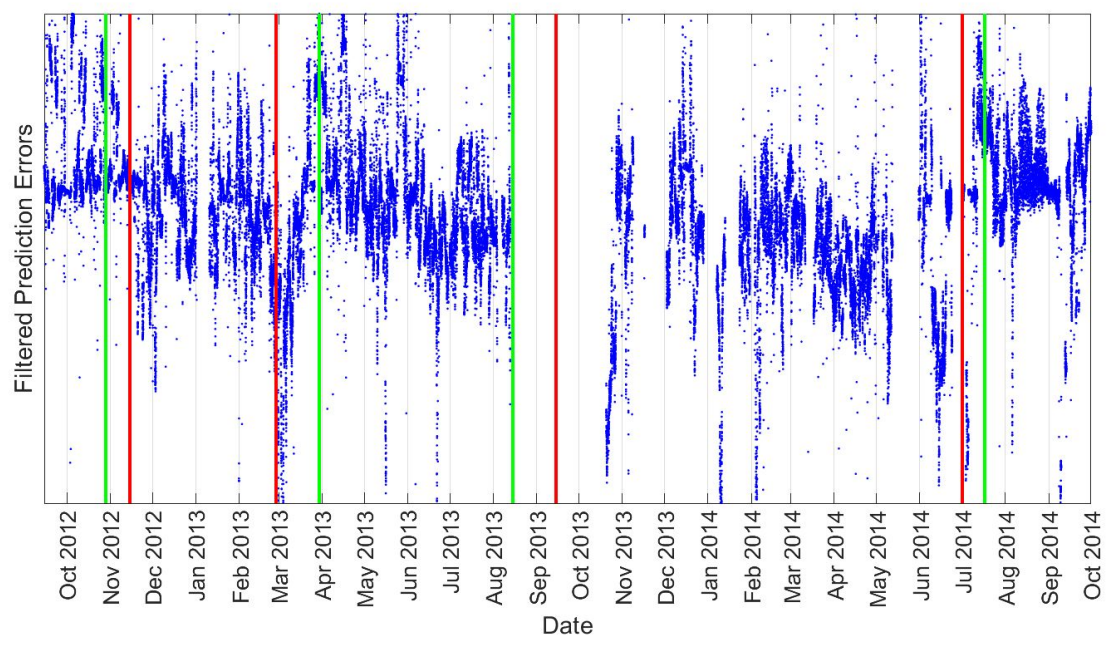

. Filtered Prediction Errors —Detected Changes — Actual Events

(b) DELM

Figure 9: Changes in time of the distribution of the percentage of speed loss estimated with the ISO 19030 and the DELM for V1. 


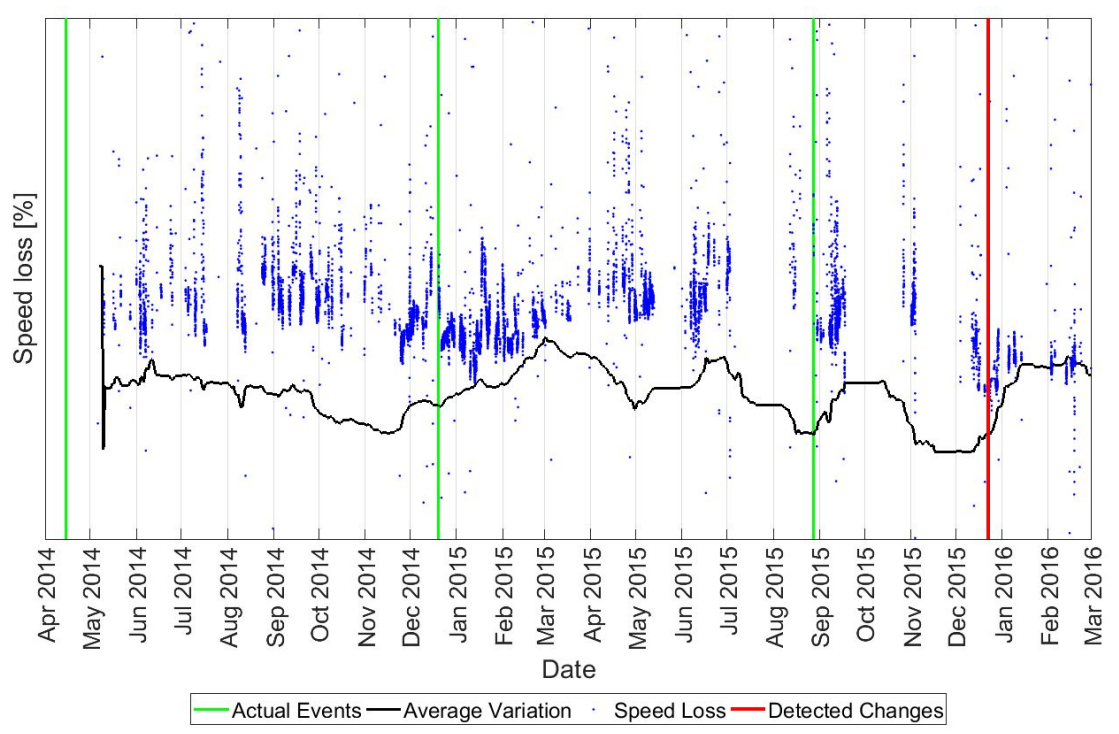

(a) ISO 19030

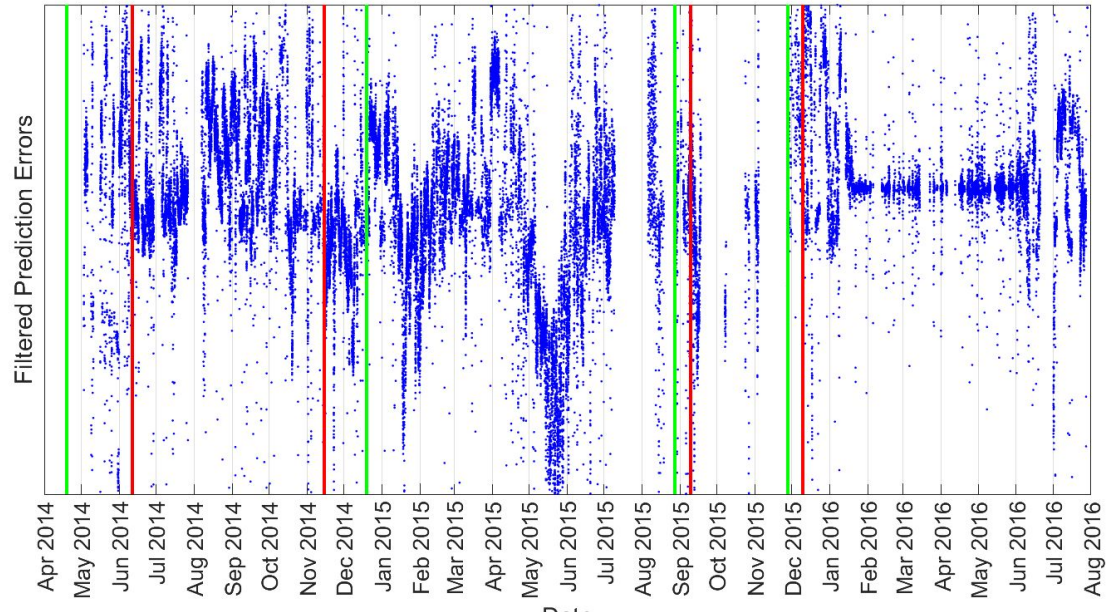

Date

Errors —Detected Changes — Actual Events

(b) DELM

Figure 10: Changes in time of the distribution of the percentage of speed loss estimated with the ISO 19030 and the DELM for V2. 
effect. Measuring the value of the speed loss before, and after, maintenance events can help understanding their effectiveness, and hence making more appropriate and informed choices. As an example, the hull cleaning performed on V2 (second maintenance event) only decreased the effect of fouling by a limited extent, while the simultaneous hull and propeller cleaning performed a few months later (third maintenance event) appeared to have a much larger effect. Similarly, the accurate estimations provided by this method could be used to evaluate the efficiency of anti-fouling paints, a widely-adopted solution to reduce the effect of fouling that, however, constitutes at the same time a cost for the company, and has a strong negative impact on the marine environment (Lindgren et al., 2016).

Given these premises, the ISO standard related to the estimation of marine fouling (ISO 15016:2015, 2015) should be integrated with the proposed methodology which, in presence of the conditions described in this paper, can lead to better results compared to the current methods. If widely adopted and associated to cleaning optimization schedules, it is possible to believe that the proposed method could significantly contribute to an increase in the operational efficiency of the global fleet, hence leading to a reduction in $\mathrm{CO}_{2}$ emissions from shipping.

\section{Conclusions}

In this work the problem of estimating the speed loss caused by the effect of fouling on the ship hull and propeller is investigated. Since marine fouling is a phenomenon that strongly affects a ship's regarding powering performance and its effects can be observed after just a few months of operations, the possibility of correctly estimate its impacts can improve the ability of the ship operators to effectively schedule the dry-docking for cleaning the hull and the propeller.

For this purpose a two-step data-driven approach, based on the Deep Extreme Learning Machines which are the most advanced tools in the context and advanced statistical methods is proposed. Thanks to such an approach, it is possible to build a Digital Twin of the ship that can be effectively exploited to detect during real operations a deviation in the speed performances (respect to the ones achievable with clean hull and propeller), and consequently to identify the extension of the marine fouling phenomena. Then the proposal has been compared with the state-of-the-art alternative method, namely the ISO 19030 standard, using real-world data coming from two Handymax chemical/product tankers. Results clearly show the effectiveness of the proposal 
and its better prediction accuracy and reliability, with respect to the ISO 19030. This is shown by both a more accurate and consistent prediction of the loss of performance over time, between cleaning intervals, and by the ability of automatically detecting maintenance events.

It has to be noted that the proposed method requires a non-indifferent amount of data in order to monitor the current speed loss, and these data need to be collected adopting an on-board network of sensors and persisted on a dedicated local storage. Moreover, since the predicted speed loss is subject to local noises, mainly due to the weather and operational conditions, the global speed loss trend has only to be seen on a wide time-span in order to understand its behaviour.

Given these premises, the application of the proposed method is beneficial both to determine the effective intervals between maintenance actions, for propeller and hull cleaning, and to estimate ship efficiency. In the future, the proposed method could be exploited also for the evaluation of the effectiveness of different energy-saving solutions, such as the case of a new propeller design or the evaluation of the benefits deriving from the application of sails. The contribution of this work can be seen as a step forward in supporting both the development of new technologies, able to improve performances and efficiency of the ship, and the implementation of suitable Condition Based Maintenance policies for increasing the shipping sustainability. Moreover, the proposed method, will facilitate the verification of the impact of new technologies or vessel components, thereby allowing to increase the transparency of energy and fuels efficiency technologies by providing a method to validate fuel savings claims made by the manufacturers and providers, supporting further uptake in the shipping industry.

\section{References}

1. IPCC, . Global warming of $1.5^{\circ} \mathrm{C}$. Tech. Rep.; Intergovernmental Panel on Climate Change; 2018. URL: http://www.ipcc.ch/report/sr15/; oCLC: 1056192590.

2. Smith, T.W.P., Jalkanen, J.P., Anderson, B.A., Corbett, J.J., Faber, J., Hanayama, S., O'Keeffe, E., Parker, S., Johansson, L., Aldous, L., et al. Third international maritime organization green house gas study. International Maritime Organization (IMO), London 2014;327. 
3. Anderson, K., Bows, A.. Executing a Scharnow turn: reconciling shipping emissions with international commitments on climate change. Carbon Management 2012;3(6):615-628.

4. Gilbert, P., Walsh, C., Traut, M., Kesieme, U., Pazouki, K., Murphy, A.. Assessment of full life-cycle air emissions of alternative shipping fuels. Journal of Cleaner Production 2018;172:855-866.

5. Lützen, M., Mikkelsen, L., Jensen, S., Rasmussen, H.. Energy efficiency of working vessels - A framework. Journal of Cleaner Production 2017;143:90-99.

6. Schim van der Loeff, W., Godar, J., Prakash, V.. A spatially explicit datadriven approach to calculating commodity-specific shipping emissions per vessel. Journal of Cleaner Production 2018;205:895-908.

7. MEPC, . Meeting Summary of the Marine Environment Protection Committee (MEPC), 72nd Session. Technical Report; Maritime Environmental Protection Committee (MEPC), part of the International Maritime Organisation (IMO); London, United Kingdom; 2018.

8. Deshpande, P.C., Kalbar, P.P., Tilwankar, A.K., Asolekar, S.R.. A novel approach to estimating resource consumption rates and emission factors for ship recycling yards in alang, india. Journal of cleaner production 2013;59:251-259.

9. Krozer, J., Mass, K., Kothuis, B.. Demonstration of environmentally sound and cost-effective shipping. Journal of Cleaner Production 2003;11(7):767777 .

10. Adland, R., Cariou, P., Jia, H., Wolff, F.. The energy efficiency effects of periodic ship hull cleaning. Journal of Cleaner Production 2018;178:1-13.

11. Calder, N.. Marine Diesel engines: Maintenance, troubleshooting, and repair. International Marine; 1992.

12. Psaraftis, H.N., Kontovas, C.A.. Ship speed optimization: Concepts, models and combined speed-routing scenarios. Transportation Research Part C: Emerging Technologies 2014;44:52-69. 
13. Demirel, Y.K., Uzun, D., Zhang, Y., Fang, H., Day, A.H., Turan, O.. Effect of barnacle fouling on ship resistance and powering. Biofouling 2017a;33(10):819-834.

14. Yebra, D., Kiil, S., Dam-Johansen, K.. Antifouling technology - past, present and future steps towards efficient and environmentally friendly antifouling coatings. Progress in Organic Coatings 2004;50(2):75-104.

15. Lindgren, F., Wilewska-Bien, M., Granhag, L., Andersson, K., Eriksson, K.. Discharges to the sea. In: Shipping and the environment: improving environmental performance in marine transportation. 2016:.

16. Schultz, M.. Frictional resistance of antifouling coating systems. Journal of fluids engineering 2004;126(6):1039-1047.

17. Kempf, G.. On the effect of roughness on the resistance of ships. Trans INA 1937;79:109-119.

18. Atlar, M., Glover, E.J., Candries, M., Mutton, R.J., Anderson, C.D.. The effect of a foul release coating on propeller performance. In: International conference on Marine Science and Technology for Environmental Sustainability (ENSUS 2002). 2002:.

19. Seo, K., Atlar, M., Goo, B.. A study on the hydrodynamic effect of biofouling on marine propeller. Journal of The Korean Society of Marine Environment \&3 Safety 2016;22(1):123-128.

20. Owen, D., Demirel, Y.K., Oguz, E., Tezdogan, T., Incecik, A.. Investigating the effect of biofouling on propeller characteristics using cfd. Ocean Engineering 2018;159:505 - 516.

21. Candries, M., Atlar, M., Anderson, C.D.. Estimating the impact of newgeneration antifoulings on ship performance: the presence of slime. Journal of Marine Engineering \&3 Technology 2003;2(1):13-22.

22. Khor, Y.S., Xiao, Q.. CFD simulations of the effects of fouling and antifouling. Ocean Engineering 2011;38(10):1065-1079.

23. Lam, J.S.L., Lai, K.H.. Developing environmental sustainability by anp-qfd approach: the case of shipping operations. Journal of Cleaner Production 2015; $105: 275-284$. 
24. Carić, H., Klobučar, G., Štambuk, A.. Ecotoxicological risk assessment of antifouling emissions in a cruise ship port. Journal of Cleaner Production 2016;121:159-168.

25. Kjaer, L.L., Pigosso, D.C., McAloone, T.C., Birkved, M.. Guidelines for evaluating the environmental performance of product/service-systems through life cycle assessment. Journal of Cleaner Production 2018;190:666678 .

26. CSC, . Air pollution and energy efficiency, a transparent and reliable hull and propeller performance standard. Tech. Rep.; Clean Shipping Coalition; 2011. URL: http://bellona.no/assets/sites/3/2015/06/fil_ MEPC_63-4-8_-_A_transparent_and_reliable_hull_and_propeller_ performance_standard_CSC1.pdf.

27. Schultz, M.P.. Effects of coating roughness and biofouling on ship resistance and powering. Biofouling 2007;23(5):331-341.

28. Atlar, M., Yeginbayeva, I., Turkmen, S., Demirel, Y., Carchen, S., Marino, A., Williams, D.. A rational approach to predicting the effect of fouling control systems on "in-service" ship performance. In: 3rd International Conference on Naval Architecture and Maritime, Yildiz Technical University, Istanbul (INT-NAM 2018). 2018:.

29. Demirel, Y.K., Turan, O., Incecik, A.. Predicting the effect of biofouling on ship resistance using cfd. Applied Ocean Research 2017b;62:100 - 118.

30. Carchen, A., Pazouki, K., Atlar, M.. A rational approach to predicting the effect of fouling control systems on "in-service" ship performance. In: Hull Performance and Insight Conference (HullPIC). 2017:.

31. Stevens, E.. The increase in frictional resistance due to the action of water on bottom paint. Naval Engineers Journal 1937;49(4):585-588.

32. ISO 19030-2. Ships and marine technology Measurement of changes in hull and propeller performance - Part 2: Default method. Standard; International Organization for Standardization; Geneva, CH; 2016.

33. Koboević, Z̆., Bebić, D., Kurtela, Z̆.. New approach to monitoring hull condition of ships as objective for selecting optimal docking period. Ships and Offshore Structures 2018;0(0):1-9. 
34. ISO 19030-1. Ships and marine technology Measurement of changes in hull and propeller performance - Part 1: General principles. Standard; International Organization for Standardization; Geneva, CH; 2016.

35. ISO 19030-3. Ships and marine technology Measurement of changes in hull and propeller performance - Part 3: Alternative methods. Standard; International Organization for Standardization; Geneva, CH; 2016.

36. Logan, K.P.. Using a ship's propeller for hull condition monitoring. Naval Engineers Journal 2012;124(1):71-87.

37. Bialystocki, N., Konovessis, D.. On the estimation of ship's fuel consumption and speed curve: A statistical approach. Journal of Ocean Engineering and Science 2016;1(2):157-166.

38. Foteinos, M., Tzanos, E., Kyrtatos, N.. Ship hull fouling estimation using shipboard measurements, models for resistance components, and shaft torque calculation using engine model. Journal of Ship Research 2017;61(2):64-74.

39. Cipollini, F., Oneto, L., Coraddu, A., Murphy, A.J., Anguita, D.. Condition-based maintenance of naval propulsion systems with supervised data analysis. Ocean Engineering 2018;149:268-278.

40. Oneto, L., Coraddu, A., Sanetti, P., Karpenko, O., Cipollini, F., Cleophas, T., Anguita, D.. Marine safety and data analytics: Vessel crash stop maneuvering performance prediction. In: International Conference on Artificial Neural Networks. 2017a:.

41. Coraddu, A., Oneto, L., Ghio, A., Savio, S., Anguita, D., Figari, M.. Machine learning approaches for improving condition-based maintenance of naval propulsion plants. Proceedings of the Institution of Mechanical Engineers, Part M: Journal of Engineering for the Maritime Environment 2016;230(1):136-153.

42. Parlak, A., Islamoglu, Y., Yasar, H., Egrisogut, A.. Application of artificial neural network to predict specific fuel consumption and exhaust temperature for a diesel engine. Applied Thermal Engineering 2006;26(8):824 828. 
43. Pedersen, B.P., Larsen, J.. Prediction of full-scale propulsion power using artificial neural networks. In: The 8th International Conference Computer and IT Applications in the Maritime Industries. 2009:.

44. Petersen, J.P., Jacobsen, D.J., Winther, O.. Statistical modelling for ship propulsion efficiency. Journal of marine science and technology 2012;17(1):30-39.

45. Radonjic, A., Vukadinovic, K.. Application of ensemble neural networks to prediction of towboat shaft power. Journal of Marine Science and Technology 2015;20(1):64-80.

46. Jonge, C.d.. Data-driven analysis of vessel performance. Master's thesis; NTNU; 2017.

47. Leifsson, L., Sævarsdóttir, H., Sigurdhsson, S., Vésteinsson, A.. Greybox modeling of an ocean vessel for operational optimization. Simulation Modelling Practice and Theory 2008;16(8):923-932.

48. Kasun, L.L.C., Zhou, H., Huang, G.B., Vong, C.M.. Representational learning with elms for big data. IEEE Intelligent Systems 2013;28(6):3134 .

49. Cambria, E., Huang, G.B.. Extreme learning machines. IEEE Intelligent Systems 2013;28(6):30-59.

50. Huang, G., Huang, G.B., Song, S., You, K.. Trends in extreme learning machines: A review. Neural Networks 2015;61:32-48.

51. Huang, G.B., Zhu, Q.Y., Siew, C.K.. Extreme learning machine: Theory and applications. Neurocomputing 2006a;70(1):489-501.

52. Huang, G.B.. An insight into extreme learning machines: random neurons, random features and kernels. Cognitive Computation 2014;6(3):376-390.

53. Huang, G.B.. What are extreme learning machines? filling the gap between frank rosenblatt's dream and john von neumann's puzzle. Cognitive Computation 2015;7(3):263-278.

54. Ridella, S., Rovetta, S., Zunino, R.. Circular backpropagation networks for classification. IEEE Transactions on Neural Networks 1997;8(1):84-97. 
55. Rumelhart, D.E., Hinton, G.E., Williams, R.J.. Learning representations by back-propagating errors. Cognitive modeling 1988;5(3):1.

56. Huang, G.B., Li, M.B., Chen, L., Siew, C.K.. Incremental extreme learning machine with fully complex hidden nodes. Neurocomputing 2008;71(4):576-583.

57. Huang, G.B., Chen, L., Siew, C.K.. Universal approximation using incremental constructive feedforward networks with random hidden nodes. IEEE Transactions on Neural Networks 2006b;17(4):879-892.

58. Huang, G.B., Zhu, Q.Y., Siew, C.K.. Extreme learning machine: a new learning scheme of feedforward neural networks. In: IEEE International Joint Conference on Neural Networks. 2004:.

59. Bengio, Y., Courville, A., Vincent, P.. Representation learning: A review and new perspectives. IEEE transactions on pattern analysis and machine intelligence 2013;35(8):1798-1828.

60. Vincent, P., Larochelle, H., Bengio, Y., Manzagol, P.A.. Extracting and composing robust features with denoising autoencoders. In: International conference on Machine learning. 2008:.

61. Zhou, H., Huang, G.B., Lin, Z., Wang, H., Soh, Y.C.. Stacked extreme learning machines. IEEE Transactions on Cybernetics 2015;45(9):20132025 .

62. Tissera, M.D., McDonnell, M.D.. Deep extreme learning machines: supervised autoencoding architecture for classification. Neurocomputing 2016;174:42-49.

63. Oneto, L., Fumeo, E., Clerico, C., Canepa, R., Papa, F., Dambra, C., Mazzino, N., D., A.. Dynamic delay predictions for large-scale railway networks: Deep and shallow extreme learning machines tuned via thresholdout. IEEE Transactions on Systems, Man and Cybernetics: Systems 2017b;47(10):2754 - 2767.

64. Glaessgen, E., Stargel, D.. The digital twin paradigm for future nasa and us air force vehicles. In: 53rd Structures, Structural Dynamics and Materials Conference. 2012:. 
65. Boschert, S., Rosen, R.. Digital twin-the simulation aspect. In: Mechatronic Futures. 2016:

66. Zhao, Y., Sun, J.. Robust truncated support vector regression. Expert Systems with Applications 2010;37(7):5126-5133.

67. Smirnov, N.V.. Approximate laws of distribution of random variables from empirical data. Uspekhi Matematicheskikh Nauk 1944;(10):179-206.

68. ISO 15016:2015. Ships and marine technology - guidelines for the assessment of speed and power performance by analysis of speed trial data. Standard; International Organization for Standardization; Geneva, CH; 2015.

69. Chauvenet, W.. A Manual of Spherical and Practical Astronomy. Lippincott; 1863.

70. Glaisher, J.W.L.. On a class of definite integrals. The London, Edinburgh, and Dublin Philosophical Magazine and Journal of Science 1871;42(280):294-302.

71. Fujiwara, T., Ueno, M., Ikeda, Y.. Cruising Performance of a Large Passenger Ship In Heavy Sea. In: The Sixteenth International Offshore and Polar Engineering Conference. 2006:.

72. Vapnik, V.N.. Statistical learning theory. Wiley New York; 1998.

73. Coraddu, A., Oneto, L., Ghio, A., Savio, S., Figari, M., Anguita, D.. Machine learning for wear forecasting of naval assets for condition-based maintenance applications. In: Electrical Systems for Aircraft, Railway, Ship Propulsion and Road Vehicles (ESARS), 2015 International Conference on. 2015:.

74. Rosasco, L., De Vito, E., Caponnetto, A., Piana, M., Verri, A.. Are loss functions all the same? Neural Computation 2004;16(5):1063-1076.

75. Caponnetto, A., De Vito, E.. Optimal rates for the regularized least-squares algorithm. Foundations of Computational Mathematics 2007;7(3):331-368.

76. Oneto, L.. Model selection and error estimation without the agonizing pain. WIREs Data Mining and Knowledge Discovery 2018;. 
77. Tibshirani, R.. Regression shrinkage and selection via the lasso. Journal of the Royal Statistical Society Series B (Methodological) 1996;58(1):267-288.

78. Zou, H., Hastie, T.. Regularization and variable selection via the elastic net. Journal of the Royal Statistical Society: Series B (Statistical Methodology) 2005;67(2):301-320.

79. Tang, J., Deng, C., Huang, G.. Extreme learning machine for multilayer perceptron. IEEE transactions on neural networks and learning systems 2016;27(4):809-821.

80. Coraddu, A., Oneto, L., Baldi, F., Anguita, D.. Vessels fuel consumption forecast and trim optimisation: A data analytics perspective. Ocean Engineering 2017;130:351-370. 